OPEN ACCESS

Edited by:

Marcello Iriti,

University of Milan, Italy

Reviewed by:

Ali Mohebbi,

Tabriz University of Medical

Sciences, Iran

Stefania Garzoli,

Sapienza University of Rome, Italy

*Correspondence:

Jorge A. M. Pereira

jorge.pereira@staff.uma.pt

Specialty section:

This article was submitted to

Food Chemistry,

a section of the journal

Frontiers in Nutrition

Received: 30 November 2021

Accepted: 26 January 2022

Published: 18 February 2022

Citation:

Pereira JAM, Casado N, Porto-Figueira $P$ and Câmara JS

(2022) The Potential of

Microextraction Techniques for the

Analysis of Bioactive Compounds in

Food. Front. Nutr. 9:825519.

doi: 10.3389/fnut.2022.825519

\section{The Potential of Microextraction Techniques for the Analysis of Bioactive Compounds in Food}

\author{
Jorge A. M. Pereira ${ }^{1 *}$, Natalia Casado ${ }^{2}$, Priscilla Porto-Figueira ${ }^{1}$ and José S. Câmara ${ }^{1,3}$ \\ ${ }^{1}$ CQM - Centro de Química da Madeira, Universidade da Madeira, Funchal, Portugal, ${ }^{2}$ Departamento de Tecnología Química \\ y Ambiental, Escuela Superior de Ciencias Experimentales y Tecnología, Universidad Rey Juan Carlos, Madrid, Spain, \\ ${ }^{3}$ Departamento de Química, Faculdade de Ciências Exatas e da Engenharia, Universidade da Madeira, Funchal, Portugal
}

For a long time, the importance of sample preparation and extraction in the analytical performance of the most diverse methodologies have been neglected. Cumbersome techniques, involving high sample and solvent volumes have been gradually miniaturized from solid-phase and liquid-liquid extractions formats and microextractions approaches are becoming the standard in different fields of research. In this context, this review is devoted to the analysis of bioactive compounds in foods using different microextraction approaches reported in the literature since 2015. But microextraction also represents an opportunity to mitigate the environmental impact of organic solvents usage, as well as lab equipment. For this reason, in the recent literature, phenolics and alkaloids extraction from fruits, medicinal herbs, juices, and coffee using different miniaturized formats of solid-phase extraction and liquid-liquid microextraction are the most popular applications. However, more ambitious analytical limits are continuously being reported and emergent sorbents based on carbon nanotubes and magnetic nanoparticles will certainly contribute to this trend. Additionally, ionic liquids and deep eutectic solvents constitute already the most recent forefront of innovation, substituting organic solvents and further improving the current microextraction approaches.

Keywords: sample extraction, microextraction by packed sorbent (MEPS), solid-phase microextraction, food analysis, liquid chromatography (LC), gas chromatography, liquid-liquid microextraction (LLME), bioactive compounds

\section{INTRODUCTION}

Foods of plant-based origin (fruits, vegetables, legumes, whole grains, spices, and beverages) constitute a natural bioresource of phytochemical secondary metabolites--polyphenols (flavonoids and non-flavonoids), vitamins, terpenes, carotenoids, capsaicinoids, glucosinolates, polyunsaturated fatty acids, among others. These metabolites exhibit powerful antioxidant (in scavenging free radicals that can easily react with nucleic acids, lipids, proteins and enzymes, causing tissue injury), anti-atherogenic, anti-inflammatory, antimicrobial, and antiproliferative properties, which protect us from degenerative diseases such as cancer $(1,2)$, aging (such as Alzheimer's and Parkinson's) (3), and cardiovascular disease $(3,4)$, longevity and assure good health (5). In addition, some of these bioactive metabolites are pigments (anthocyanins, flavones, carotenoids) which confer the typical color of foods and therefore can be isolated to be used as colorants in the food industry instead of the potentially harmful artificial colorants (butylated 
hydroxytoluene, tert-butyl hydroquinone, and butylated hydroxyanisole). In turn, the antioxidants are used to improve and increase the preservation, food shelf life, food quality and safety, and more recently as active ingredients in smart food packaging. Apart from the above mentioned field, secondary metabolites are also extensively being used in (i) pharmaceutical (antioxidant, anti-inflammatory and neuroprotective agents); (ii) cosmetic industry, as sun protectors, antiaging, antiinflammatory and healing agents; and (iii) environmental applications as metal chelators, natural dyes (theaflavin) and biosorbents (anthocyanins- and tannins-based) (6). In recent years, scientific and industrial interest in bioactive compounds has increased considerably, supported by the growing and growing demand of consumers for foods that promote health and prevent certain diseases, namely those etiologically related to oxidative stress (7). These metabolites are not fundamental for the organism's vital processes, for their development and growth, but they can act as modulators of several important biological activities and functions giving them preventive properties of certain age-related pathologies and metabolic disorders.

Polyphenols-These secondary metabolites are notably recognized by their antioxidant activity. In plants, polyphenols stand out for their physiological and morphological importance, being used as a defense mechanism against oxidative stress, preventing damage to the DNA, protecting plants from UV radiation and aggression by parasites and predators (8). However, the enormous interest that these secondary metabolites have aroused in the international scientific community is related not only to their potential industrial applications but due to their beneficial effects on human health. Indeed, significant preventive potential in the development of chronic diseases mediated by oxidative stress including cardiovascular diseases (CVDs), cancer, type 2 diabetes mellitus, obesity, and neurodegenerative diseases (NDDs), is associated with polyphenols (8-10). According to chemical structure, polyphenols can be grouped into two categories: flavonoids and non-flavonoids. Flavonoids constitute the most important and abundant group, having a common structure of C6-C3-C6 diphenylpropanes consisting of 2 aromatic rings normally linked by an oxygenated heterocycle of 3 carbon atoms (11). This group of polyphenols can be subdivided into several subclasses represented by flavonols, flavones, flavanones, isoflavones, flavonoids and anthocyanins. The non-flavonoids group include as main subclasses phenolic acids, stilbenes, lignans and tannins.

Biogenetically, the synthesis of polyphenols involves a relatively complex network of secondary metabolic pathways that include the phenylpropanoid pathways through which phenolic acids are synthesized and the xiquimic acid pathway, where mainly flavonoids, tannins and stilbenes are formed.

The main precursors are the phenylalanine from the shikimate pathway and malonyl-CoA from the acetate pathway.

Vitamins - are found in a wide variety of fruits and vegetables, are reported as powerful dietary antioxidants with the potential to prevent heart, chronic inflammatory and neurodegenerative diseases (12). It has been shown that vitamin C could inhibit the oxidation of the complex formed between the low-density lipoproteins (LDLs) and proteins, contributing to reducing atherosclerosis, conserving the cardioprotective properties. Vitamin E prevents HDL from lipid oxidation and protects LDLs, DNA, and polyunsaturated fatty acids from oxidative damage. It, moreover, plays a role in the modulation of the immune response, hemoglobin biosynthesis, and stabilization of the structure of membranes.

Carotenoids-Located in subcellular organelles (plastids), mainly associated with proteins in the chloroplasts, carotenoids are naturally occurring pigments found in plants, fungi, algae, and bacteria, generally C40 tetraterpenes/tetraterpenoids formed from eight C5 isoprenoid units $(13,14)$. Consumption of carotenoids has been associated with a range of functions in human health, including a reduced risk of age-related macular degeneration and cataract (15). In addition to beneficial effects on eye health, by absorbing specific wavelengths of light, carotenoids also produce improvements in cognitive function and cardiovascular health, by blocking the formation and oxidation of LDLs, and may help to prevent some types of cancer, by limiting the abnormal growth of cells $(16,17)$. The most abundant carotenoid found in human serum is lycopene (carotenoid pigment responsible for the red color), found in high amounts in tomatoes, recognized as the most effective antioxidant among all the carotenoids.

Glucosinolates-are a large group of sulfur- and nitrogencontaining glucosides, which are abundant plant secondary metabolites, with nutritional effects and biologically active compounds found principally in the plant order Brassicales and related families of vegetables like broccoli, Brussels sprouts, and kale, which impart a characteristic pungent aroma and bitter taste. Therefore, they are frequently consumed as a normal part of the human diet $(18,19)$. Scientific studies support that consumption of glucosinolates, and their derivatives are associated with a preventive anticancer effect, namely of the lungs and alimentary tract, together with increased activity of detoxifying enzymes and resultant decreases in DNA damage (20).

\section{Bioactive Compounds in Disease Prevention}

Plant-based foods rich in bioactive compounds could be used as functional ingredients for providing many health benefits. Several epidemiological studies related unhealthy dietary behaviors (malnutrition, tobacco, alcohol, physical inactivity) with chronic diseases such as cancer, type 2 diabetes, and cardiovascular diseases, which are leading causes of death in developed countries.

A diet rich in bioactive compounds, namely by the consumption of fruits, vegetables/legumes and spices, has a preventive action in the development of neurodegenerative diseases due to its ability to influence and modulate various cellular processes, such as signaling, proliferation, apoptosis, redox balance and differentiation $(21,22)$. It was demonstrated by Sarubbo et al. (23) that anthocyanins improve the function of hippocampal-dependent memory, preventing neurodegeneration by decreased levels of inflammatory biomarkers such as NF-kB, TNF- $\alpha$, IL- $1 \beta$, ROS, and RNS, 
reducing neuronal apoptosis through Bax, cytochrome c, caspase 3 and PARP-1 and increasing the levels of proteins such as Akt, GSK3 $\beta$ and BCL-2. Specifically, apigenin, one of the major polyphenolics in citrus fruit, parsley and celery, and quercetin, which is abundantly found in onions, green tea and apples, showed a potent anti-aggregating $\beta$-amyloid $(\mathrm{A} \beta)$ activity reducing the formation of amyloid plaques that are at the origin of the symptoms associated with Alzheimer's disease (23).

Moreover, some bioactive compounds can act in different phases of the cell cycle, repairing DNA damage and promoting cell apoptosis of the damaged cells to prevent carcinogenesis (24). Some bioactive compounds interfere with cancer initiation, promotion, and progression steps, modulating different enzymes and receptors in signal transduction pathways related to cell proliferation, differentiation, apoptosis, inflammation, angiogenesis, and metastasis. In addition, they also exert their preventive effect in decreasing resistance to multiple therapies and help regulate hormones and growth factors. They prevent DNA oxidative damage and modulate the immune system response (25). When combined with therapeutic agents such as Paclitaxel and Doxorubicin, some of the polyphenols allow for a decrease in the dose of the drug, thus decreasing the side effects of chemotherapy. The bioactive compounds are also associated with protective effects on the cardiovascular system (26). In addition to their antioxidant and anti-inflammatory activity, several mechanisms evidence these effects, namely those related to the lowering of blood pressure, the improvement of endothelial function, the inhibition of platelet aggregation and LDLs oxidation, also related to the prevention of the formation of atherosclerotic lesions, and with the enzymatic modulation expressed, for example, in the regulation of redox enzymes, thus contributing to reducing the risk of cardiovascular diseases. Flavonoids and catechins are the polyphenols that exert the most significant effect in reducing the risk of cardiovascular incidents. The flavonoids help to delay the development of atherosclerotic plaque and atherosclerosis by reducing endothelial dysfunction. In addition, flavonoids will interact with the membrane modifying its fluidity. Catechins help in smooth blood circulation since they can reduce the accumulation of cholesterol and its oxidation products in the walls of arteries. Moreover, they function as antiatherogenic agents by blocking oxidized LDLs-induced endothelial apoptosis. Figure 1 provides an simplified overview of the main classes of food bioactive compounds and targeted diseases. Several in vitro and in vivo assays have been developed to evaluate the antioxidant activity of several plant-based foods rich in bioactive compounds $(27,28)$. These assays have demonstrated the importance of secondary metabolites such as phenols, flavonoids, carotenes, among others, with verified antioxidant activity for the prevention of degenerative disorders. The most common in vitro assays include 2,2-diphenyl-1-picrylhydrazyl radical (DPPH) assay; total radical trapping antioxidant parameter (TRAP) assay; oxygen radical absorbance capacity assay (ORAC); reducing power assay; ferric reducing-antioxidant power (FRAP); superoxide radical scavenging activity, determination of phenol content by the Folin-Ciocalteu method; and total flavonoid content. The in vivo assays include total flavonoid content; reduced glutathione
(GSH) assay; glutathione peroxidase (GPx) assay; glutathioneS-transferase (GSt) assay; glutathione reductase (GR) assay; and superoxide dismutase (SOD) assay. A huge amount of highresolution analytical methodologies, from chromatographic techniques $(29,30)$ to nanobiosensors $(31,32)$ constitute powerful analytical tools that might be applied in food analysis, able to cope with a large number of analytes in food matrices, including those responsible for bioactive potential. The selection of the analytical technique depends mainly on the chemical nature, properties and required robustness and sensitivity of the target analytes to be determined.

\section{SAMPLES EXTRACTION IN FOOD ANALYSIS \\ Importance of Sample Extraction in Food Samples}

Food samples are extremely complex matrices, as they present a wide variety of compounds in their composition, such as proteins, fats, sugars, salts, vitamins, additives, etc., with different polarity, size and chemical properties. For this reason, the determination of a specific bioactive compound in a food sample is not an easy task, since its extraction and detection can be hampered due to the interferences caused by the rest of the components present in the food matrix (33). These matrix interferences usually have a negative impact on important analytical parameters, such as the limits of detection and quantification, the accuracy, and the precision, leading to low sensitivity and selectivity in the analysis (34). Consequently, food samples, in general, cannot be analyzed directly. Instead, they must undergo a proper extraction procedure before the instrumental analysis. Therefore, in food analysis, it is of utmost importance to carry out a sample extraction that enables cleanup, selective extraction, purification and even pre-concentration of the target analytes, to facilitate and enhance their following instrumental analysis (35) (Figure 2). Indeed, sample extraction is one of the most critical steps of any sample preparation technique. The main aim of sample extraction is to minimize the sample complexity and eliminate most matrix interferences. This can be obtained through the isolation, purification and pre-concentration of the analytes of interest in a matrix, while simultaneously turning them into a form compatible with the instrument used for their subsequent analysis. This step often involves a chromatographic technique (e.g., high performance liquid chromatography - HPLC, or gas chromatography, GC) coupled to a powerful detector (normally mass spectrometry (MS) or ultraviolet (UV) detectors) (36). The extraction of a specific analyte depends on several parameters, like its solubility, the mass transfer between the sample and the solvent, and the matrix interferences. Moreover, the nature of the sample also plays an important role. In this sense, liquid food samples can be directly extracted with a suitable solvent. However, solid food samples require a previous preparation step before extraction to improve the efficiency of the process, which involves reduction of the sample particle size (grinding) for better mass transfer and, normally, a drying step to decrease the moisture (as it may reduce 


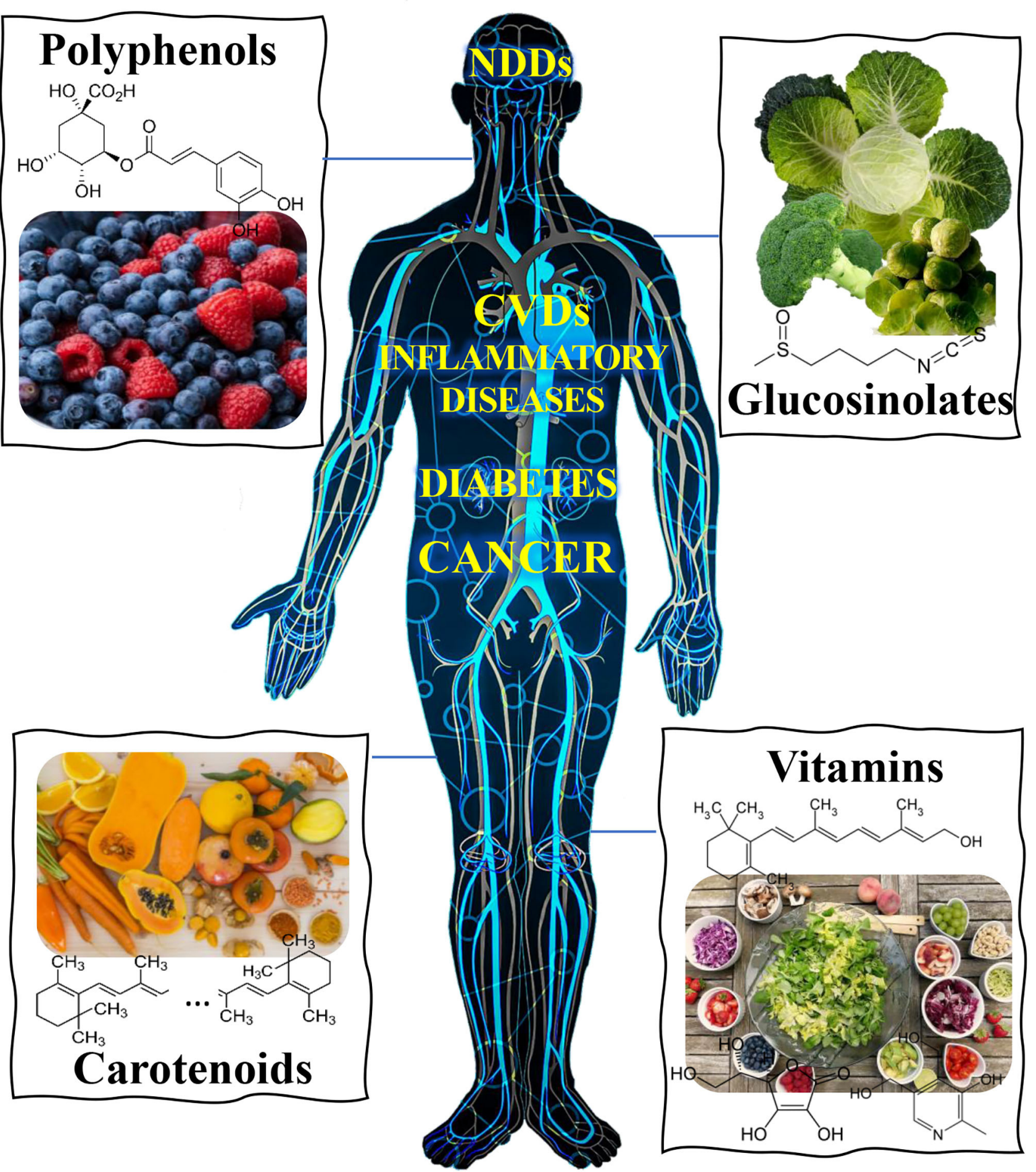

FIGURE 1 | Overview of main classes of food bioactive compounds and reported activity against different human diseases, namely neurodegenerative (NDDs), cardiovascular (CVDs) and inflammatory diseases, cancer, diabetes, etc.

the extraction efficiency). Often freeze drying, or lyophilization, is applied to solid samples before grinding, particularly when they present high levels of water content. These procedures are also important to homogenize the sample and ensure that each aliquot is representative of the whole sample composition, therefore avoiding biased results of food composition in the analyte of interest. As we will discuss below, as extraction approaches are evolving to sophisticated microextraction procedures requiring 


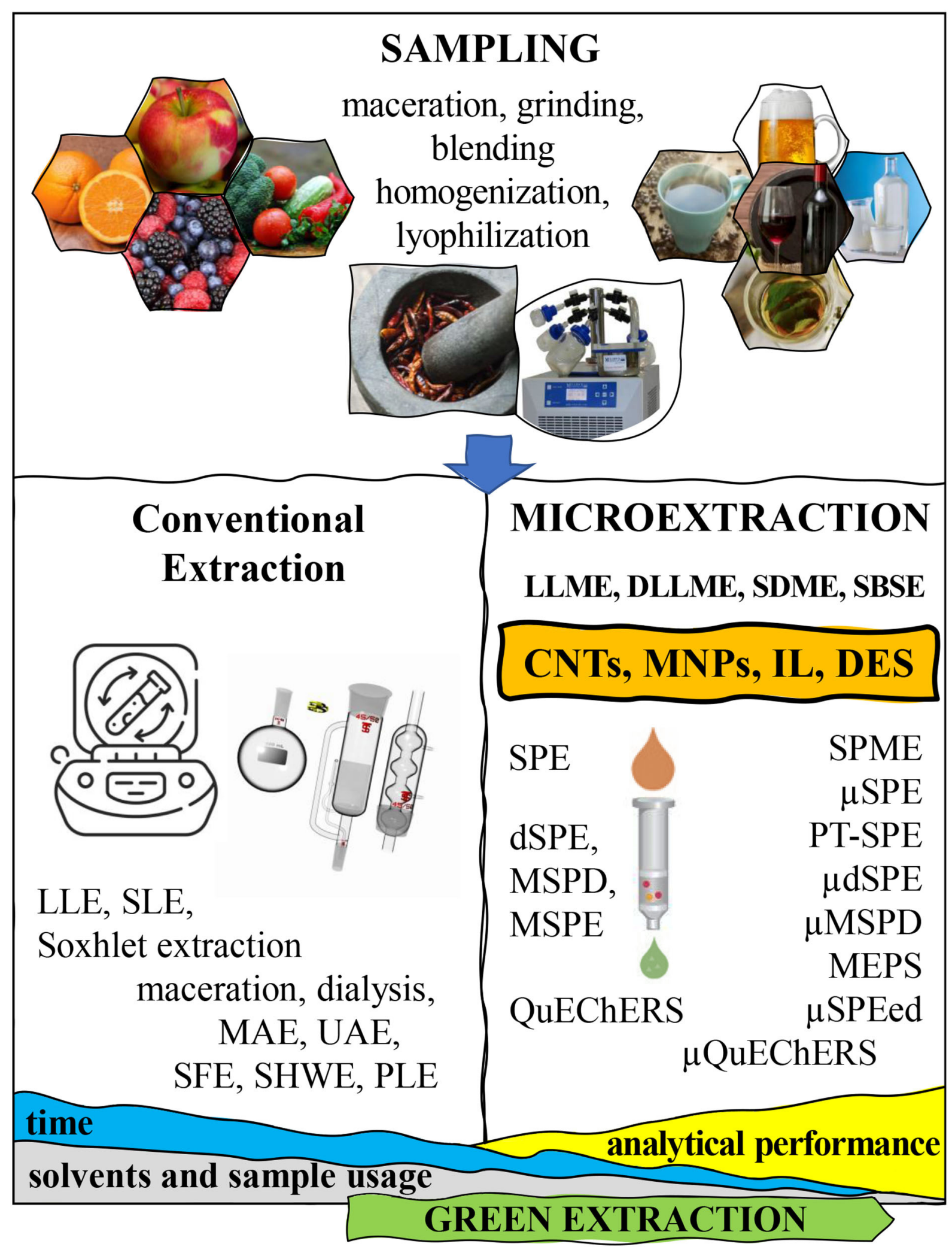

FIGURE 2 | Overview of the most relevant steps in the extraction layout of food samples, from sampling to microextraction of the target analytes. 
progressively lower sample amounts, sample homogenization is becoming critical to obtain reproducible results. After these operations, the solid powder can be extracted with a suitable solvent (solid-liquid extraction, SLE) obtaining a sample extract solution that can further be treated as a liquid sample (37) (Figure 2).

\section{Conventional Sample Extraction Approaches}

Solvent-based extraction techniques, such as liquid-liquid extraction (LLE), Soxhlet extraction, maceration, membrane extraction (dialysis), microwave-assisted extraction (MAE), ultrasound-assisted extraction (UAE), supercritical fluid extraction (SFE), super-heated water extraction (SHWE) and pressurized liquid extraction (PLE), have been traditionally applied to the extraction of bioactive compounds from food samples (37-39). These techniques are based on the contact of the sample (liquid or solid) with a suitable solvent. However, these solvent-based techniques do not achieve selective extraction of the analytes, besides providing insufficient clean-up of the samples. For these reasons, in the last decades, the combination of solvent-based procedures with sorbent-based extraction techniques has aroused special interest in the extraction of food samples, due to the higher extraction capacity and selectivity achieved (40). The sorbent-based extraction techniques are based on sorption processes that enable the selective extraction of the analytes from the matrix by their retention in a sorbent material with specific and related characteristics, allowing the purification and concentration of the analytes at the same time. Accordingly, in the extraction of food samples, there is usually a first step involving the contact of the sample with an organic solvent under different conditions (pressurized, assisted, etc.) to obtain a sample extract, which is afterwards purified in the next step by a suitable sorbent-based extraction technique. In the case of liquid samples, they can be directly purified with the sorbent material. In this context, solid-phase extraction (SPE) is the main sorbent-based extraction technique and has become the extraction procedure of choice in the food field, replacing the classic LLE, due to its selectivity and versatility (37). Moreover, the great variety of sorbent materials available to perform sorbent-based extractions (e.g., reversed-phase, ion-exchange, mixed-mode, molecularly imprinted polymers, magnetic nanoparticles (MNPs), multiwalled carbon nanotubes (MWCNTs), metal-organic frameworks, etc.) offers a wide range of possibilities to achieve selective extraction of analytes with different chemical properties. This has opened in the last years new research lines focused on the development of new sorbent materials designed with specific characteristics depending on the type of analytes, so a better extraction efficiency and suitable clean-up can be achieved (33-36, 40-42).

\section{Different Solvent- and Sorbent-Based Formats for the Extraction of Food Samples}

For many years, the conventional extraction techniques LLE and SPE have been popular choices for the extraction of food samples. However, LLE presents some inherent drawbacks, such as large volumes of organic solvents, limited ability to extract polar compounds, long time consumption, etc. Consequently, SPE is preferred in food analysis, as it provides better extraction efficiency and requires less consumption of organic solvents than LLE (43). Nevertheless, since the first appearance of the SPE, other sorbent-based procedures based on the SPE extraction principle have been proposed over the last decades, such as dispersive SPE (dSPE), matrix solid-phase dispersion (MSPD) and magnetic SPE (M-SPE). The difference between the SPE and these techniques lies basically in how the sorption process between the analytes and the sorbent is carried out. For instance, in SPE and MSPD the sorbent material is packed in an extraction cartridge, whereas in dSPE and MSPE the sorbent is dispersed in the sample solution. In the case of MSPD, the sorbent is previously dispersed and blended with the sample, and then the mixture is packed in the cartridge, while in SPE only the sorbent is packed in the cartridge. On the other hand, any type of sorbent can be used in dSPE, but in MSPE, only MNPs can be employed. In general, these sorbent-based techniques arose to improve SPE, by reducing the number of steps, decreasing the volume of organic sorbents, enhancing the sorbent-analyte interaction, and making easier the extraction procedure $(33,41,42)$.

\section{EMERGENT MICROEXTRACTION APPROACHES FOR FOOD ANALYSIS}

The current trend in sample extraction is to perform sustainable procedures that fulfill the Green Analytical Chemistry (GAC) requirements, which mainly involve using minimal amounts of solvents and samples, carrying out fewer steps in the procedures and reducing the waste generation $(43,44)$. This way, more costeffective and environmentally friendly extraction procedures can be achieved. This can be accomplished by the miniaturization of the extraction procedures. Accordingly, the conventional sorbent-based extraction procedures have been scaled down and applied to the extraction of bioactive compounds in food samples. For instance, the miniaturization of SPE has been carried out for the extraction of flavanones from citrus fruits (45), miniaturized dSPE has been applied to the extraction of pcoumaric acid and p-hydroxybenzoic acid from fruit juices (46), miniaturized MSPD was used to extract different polyphenols from olive fruits (47) and flavonoids have recently been extracted from different food items by miniaturized dSPE (48). Likewise, the QuEChERS (Quick, Easy, Cheap, Effective, Rugged and Safe) strategy first developed in 2003 (49), which involves simultaneous extraction and clean-up of samples, has also been successfully miniaturized and applied to the extraction of 12 polyphenols in baby foods (50). Nonetheless, in the past decades, several microextraction methods based on SPE and LLE have emerged with new configurations and formats intending to improve the drawbacks of conventional extraction procedures and address some key challenges, such as the need to use lower sample volumes and perform quicker analysis. In this sense, LLE has been the axis to develop different solvent-based microextraction methods, including liquid-liquid microextraction (LLME), 
dispersive liquid-liquid microextraction (DLLME), single-drop microextraction (SDME), liquid-liquid-liquid microextraction (LLLME) and hollow-fiber liquid-phase microextraction (HFLPME). On the other hand, sorbent-based extraction techniques have evolved beyond the SPE principle, leading to the design and development of more innovative extraction formats and devices, such as solid-phase microextraction (SPME), stir-bar sorptive extraction (SBSE), microextraction by packed sorbent (MEPS), $\mu$ SPEed ${ }^{\circledR}$ and pipette-tip SPE (PT-SPE). These microextraction techniques are increasingly being proposed as an alternative to conventional extraction procedures in food analysis, as they enable "greener" approaches that meet the GAC requirements. Figure 2 offers an overview of the context of microextraction as an evolution from sampling to emergent microextraction approaches. To organize their discussion, selected examples of the analysis of bioactive compounds in foods involving microextraction were primarily divided according to the physical state of the sorbent used in the retention of the target analytes. This results, in a simplistic way, in microextraction approaches using solid sorbents, like exposed solid sorbents (SPME), packed sorbent particles ( $\mu$ SPE, pipette-tips SPE, MEPS, $\mu$ SPEed), or dispersed sorbent particles (QuEChERS, dSPE, MSPD), described in more details in Table 1. In turn, Table 2 is devoted to applications involving a liquid sorbent, like different formats of miniaturized LLE ( $\mu$ LLME, $\mu$ DLLME, $\mu$ SDME). The use of ionic liquids (ILs) and deep eutectic solvents (DES) as extraction solvents is being successfully applied to different formats of microextraction and will be also discussed.

\section{Solid-Phase Microextraction and Related Formats}

SPME is certainly one of the most popular extraction methodologies and despite it has been introduced three decades ago, it continues to be an important reference in what concerns microextraction approaches. Often, its usage in food analysis relates to flavor composition, namely the characterization of the volatiles organic compounds (VOCs) that define the aroma of different fruits, like mangoes (51) and tangerines (52). Many of these VOCs are bioactive compounds with promising properties, as thymol identified in tangerines cultivated in Madeira Island (52). The same authors used a related methodology, needletrap microextraction (NTME) to identify many bioactive VOCs in lemons also grown in the same geography (56). Unlike SPME, NTME is an exhaustive approach that allows a deeper and broader coverage of the volatile composition of different samples, exhibiting improved detection limits and reduced sampling time and volume (117). Moreover, this format allows sampling and storage in the same device (needle trap) for much longer times than SPME, from days to weeks, depending on the type of the target VOCs (117). This makes NTME suitable for sampling procedures distant from the laboratory facilities where the gas chromatography mass spectrometry (GC-MS) analysis is performed. But in contrast to NTME, SPME has been also used to analyze non-volatile bioactive compounds, as phenolics in juices and wines $(54,55)$. Related with this, Shou and collaborators have been proposed different approaches merging pipette tip microextraction with SPME (PT-SPME) for the quantification of important bioactive flavonoids and alkaloids in different Traditional Chinese Medicine preparations $(53,57)$. The comparisons made with conventional extractions approaches LE and SPE, clearly point to the potential of the methodologies proposed, namely in what concerns to the higher analytical performance they allow (Table 1). Phenolic compounds are one of the most widespread groups of bioactive compounds and different SPME approaches have been also used to quantify several of these compounds in wines and fruit juices $(54,55,118)$ (Table 2).

\section{Micro Solid-Phase Extraction and Related Formats}

Taking into consideration the success and widespread use of SPE, its miniaturization and downscale is a logical consequence of the adoption of GAC requirements, namely in what concerns the usage of organic solvents, which are greatly reduced in the transition from SPE to $\mu$ SPE. Moreover, the ease of use, time of extraction, availability, and automatization potential, contribute to the success of $\mu$ SPE as well as different approaches developed. In the traditional concept of $\mu \mathrm{SPE}$ using a sorbent entailed between two fritzes in a syringe tube, as well as in the PTSPE, that uses instead a pipette tip, new sorbent particles have been successfully reported, for instance, to extract gallic acid (GA). Also known as 3,4,5-trihydroxybenzoic acid, this is a low molecular triphenolic compound widely present in different plants and able to elicit a myriad of bioactive effects in the human body [reviewed in Choubey et al. (119)]. For this reason, its quantification in foodstuffs is very important and, in this aspect, the microextraction approaches reported by Hao et al. (58) and Arabi et al. (61) are very relevant. Hao et al. (58) developed a novel surface imprinting polymer based on magnetic carbon nanotubes (M-CNTs) to extract GA from pomegranate rinds samples, while Arabi et al. (61) reported the use of pipette tip SPME (PT-SPME) using molecularly imprinted silica monolithics to extract the same phenolic acid from juices. Related to this, Khajeh et al. (116) proposed an electrokinetic approach for the simultaneous extraction of GA and caffeine from coffee which is very promising but needs further improvement to lower the extraction times to acceptable values ( $50 \mathrm{~h}$ to achieve $95 \%$ extraction, only $20 \%$ target analytes extraction after $5 \mathrm{~h}$ ). The use of molecular imprints obtained against specific analytes elicits more efficient extractions, as reported for estradiol extraction from milk using a PT-SPE approach (62) (Table 2). Another type of sorbent particle, the mesoporous molecular sieve Santa Barbara Amorphous 15 (SBA-15), has been also used with success in $\mu$ SPEs formats to extract flavanones from citrus fruits (45) and triterpenoid saponins from TCM (63). MEPS and $\mu$ SPEed formats are very interesting forms of SPE miniaturization in which the sorbent particles are tightly packed inside a BIN (MEPS) or cartridge ( $\mu$ SPEed) and operated in different formats, including hand-held automatic syringes able to control the solvent flow. This allows pressure-driven extractions which are considerably more efficient than conventional SPEs [reviewed in Pereira et al. (120)]. The more recent $\mu$ SPEed approach uses 
TABLE 1 | Selected examples of microextraction of bioactive compounds using solid sorbents.

\begin{tabular}{|c|c|c|c|}
\hline $\begin{array}{l}\text { Extraction } \\
\text { format/methodology }\end{array}$ & $\begin{array}{l}\text { Target } \\
\text { analytes/sample }\end{array}$ & Method description/analytical performance (LOD) & References \\
\hline HS-SPME & VOCs/mangoes & $\begin{array}{l}\text { Blended } 5.0 \mathrm{~g} \text { juice }+1.5 \mathrm{~g} \text { of } \mathrm{NaCl} \text { in } 15 \mathrm{~mL} \text { capped vial, } 80 \mathrm{rpm} \text { stirring; } 10 \\
\text { min-equilibration; } 30 \mathrm{~min} \text { DVB/Carboxen/PDMS extraction }\left(40^{\circ} \mathrm{C}\right) \text {; GC desorption }\end{array}$ & $(51)$ \\
\hline HS-SPME & VOCs/tangerines & $\begin{array}{l}5 \mathrm{~mL} \text { tangerine juice or } 250 \mathrm{mg} \text { peels }(20 \mathrm{~mL} \mathrm{HS} \text { glass vial, magnetic stirring); } \mathrm{NaCl} \\
10 \%(\mathrm{w} / \mathrm{v}) ; \mathrm{HS}-\mathrm{SPME} \text { extraction }\left(40 \mathrm{~min}, 40^{\circ} \mathrm{C}\right) ; \mathrm{GC}-\mathrm{MS} \text { analysis }\end{array}$ & $(52)$ \\
\hline $\begin{array}{l}\text { EPT-SPME/PT- } \\
\text { SPME }\end{array}$ & $\begin{array}{l}\text { Alkaloids, } \\
\text { flavonoids/TCM }\end{array}$ & $\begin{array}{l}\text { Sample pretreatment: liquid samples }(1.0 \mathrm{~mL}) \text { centrifugation }(5,000 \mathrm{~g}, 10 \mathrm{~min}, \mathrm{RT}) \text {; } \\
\text { EPT-SPME: hollow polypropylene fiber attached to } 1,000 \mu \mathrm{L} \text { pipette tip; } 10 \mathrm{mg} \\
\text { sorbent }+2 \mathrm{mg} \text { sodium bicarbonate placed in the tip; } 500 \mu \mathrm{L} \text { sample supernatant + } \\
100 \mu \mathrm{L} \text { sodium dihydrogen phosphate }\left(0.5 \mathrm{~mol} \mathrm{~L}^{-1}\right) \text { loaded into the tip; } 1.2 \mathrm{~min} \\
\text { dwelling; sample withdrawn; washing }(200 \mu \mathrm{L} \text { water); desorption ( } 100 \mu \mathrm{L} \\
\text { MeOH-ammonia (90:10, v/v) withdrawn into the tip; } 1.5 \text { min dwelling; solvent } \\
\text { withdraw; UHPLC-UV analysis ( } 20 \mu \mathrm{L}) \text {. PT-SPME: } 10 \mathrm{mg} \text { sorbent placed in the tip; } \\
\left.500 \mu \mathrm{L} \text { sample supernatant }+100 \mu \mathrm{L} \text { phosphate buffer (0.5 mol } \mathrm{L}^{-1}, \mathrm{pH} 6.5\right) \text { loaded } \\
\text { into the tip; } 1.2 \text { min dwelling; } 200 \mu \mathrm{L} \text { water washing; desorption: } 100 \mu \mathrm{L} \mathrm{MeOH} \text { : } \\
\text { ammonia }(90: 10, \mathrm{v} / \mathrm{v}) \text { withdrawn into the tip; } 1.5 \text { min dwelling; UHPLC } \\
\text { analysis/1.02-2.98 } \mu \mathrm{gL}^{-1}\end{array}$ & $(53)$ \\
\hline HS-SPME & $\begin{array}{l}\text { Trans- } \\
\text { resveratrol/wines, } \\
\text { grape juices }\end{array}$ & $\begin{array}{l}30 \text { min extraction (1.5-mL amber vials; HS-SPME with magnetic stirring; } 6.6 \% \text { EtOH } \\
+10 \% \mathrm{NaCl} \text { ); } 15 \text { min fiber soaking (mobile phase); desorption; LC-UV analysis/0.4 ng } \\
\mathrm{mL}^{-1}\end{array}$ & $(54)$ \\
\hline MMF-SPME & $\begin{array}{l}\text { Phenolic acids/fruit } \\
\text { juices }\end{array}$ & $\begin{array}{l}\mathrm{MeOH}+\text { water activation; } 20 \mathrm{~mL} \text { sample }(25 \mathrm{~mL} \text { vial }+ \text { stirring bar, } 8 \times 2 \mathrm{~mm}) \text {; MMF } \\
\text { direct immersion in sample solution; } 40 \mathrm{~min} \text {, low stirring; } \mathrm{MeOH} / 1 \% \mathrm{AA} \text { aqueous }(\mathrm{v} / \mathrm{v} \text {, } \\
1 / 490 / 10 \text { ) desorption }(400 \mu \mathrm{L}, 20 \mathrm{~min}) / 0.17-0.57 \mu \mathrm{L}^{-1}\end{array}$ & $(55)$ \\
\hline NTME & VOCs/lemon & $\begin{array}{l}250 \mathrm{mg} \text { sample ( } 20 \mathrm{ml} \mathrm{HS} \text { glass vial), equilibration }\left(10 \mathrm{~min}, 50^{\circ} \mathrm{C}\right) ; 30 \mathrm{~mL} \text { gas phase } \\
\text { (30 cycles) loaded through the NTD (pre-attached to } 1 \mathrm{~mL} \text { syringe); GC-MS analysis }\end{array}$ & $(56)$ \\
\hline PT-SPE & Flavonoids/TCM & $\begin{array}{l}100 \mu \mathrm{L} \text { sample loaded into tip ( } 5 \mu \mathrm{L} \mathrm{s}^{-1} \text { flow rate); } 1.0 \text { min dwelling; } 500 \mu \mathrm{L} \text { water } \\
\text { washing; } 100 \mu \mathrm{L} \text { ACN desorption ( } 5 \mathrm{~min} \text { dwelling); HPLC analysis }(20 \mu \mathrm{L}) \text {. SPE: } 500 \\
\mu \mathrm{L} \text { sample loaded (positive pressure) onto preconditioned Sep-Pac C18-360 mg } \\
\text { cartridge ( } 3.0 \mathrm{~mL} \mathrm{MeOH}+3.0 \mathrm{~mL} \text { water); } 2 \times 5 \mathrm{~mL} \text { water washing; } 3.0 \mathrm{~mL} \text { ACN } \\
\text { elution; evaporation to dryness }\left(40^{\circ} \mathrm{C}, \mathrm{N}_{2} \text { stream); } 100 \mu \mathrm{L} \text { ACN reconstitution, vortex, }\right. \\
\text { and centrifugation (10,000 rpm, } 10 \mathrm{~min}) ; \mathrm{HPLC}-\mathrm{UV} \text { analysis }(2 \mu \mathrm{L}) / 0.027-0.045 \mu \mathrm{g} \\
\mathrm{mL}^{-1}\end{array}$ & $(57)$ \\
\hline M-MWCNTS-SPE & GA/pomegranate rind & 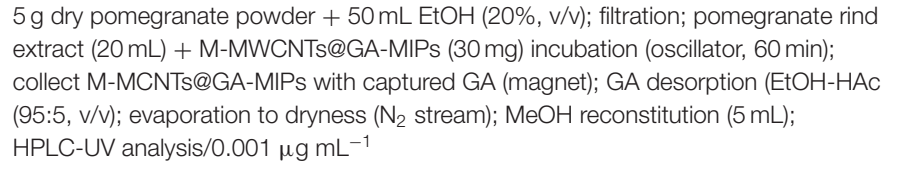 & $(58)$ \\
\hline M-MIP-SPE & $\begin{array}{l}\text { Chlorogenic acid/ fruit } \\
\text { juices }\end{array}$ & $\begin{array}{l}30 \mathrm{~mL} \text { fruit juice }+60 \mathrm{mg} \mathrm{Fe}_{3} \mathrm{O}_{4} @ \mathrm{CGA}-\mathrm{MIPs} ; 30 \mathrm{~min}, \mathrm{RT} ; \mathrm{Fe}_{3} \mathrm{O}_{4} @ \mathrm{CGA}-\mathrm{MIPs} \\
\text { separated magnetically, EtOH-HAc }(95: 5, \mathrm{v} / \mathrm{v}) \text { elution }(6 \mathrm{~h}) \text {; filtration }(0.22 \mu \mathrm{m} \text { nylon } \\
\text { membrane); HPLC-UV analysis/0.01 } \mu \mathrm{g} \mathrm{mL}^{-1}\end{array}$ & $(59)$ \\
\hline M-SPE & Flavonoids/ tea, wine & $\begin{array}{l}2 \mathrm{~mL} \text { samples into a centrifuge tube; } 8 \mathrm{mg} \text { magnetic graphene composites } \\
\left(\mathrm{GO} / \mathrm{Fe}_{3} \mathrm{O}_{4}\right) ; 2 \mathrm{~mL} \text { phosphate buffered solution }(0.02 \mathrm{M}) \text {; sonication }(5 \mathrm{~min}) \text {, vortex } \\
\text { ( } 15 \mathrm{~min} \text { in an oscillator); remove supernatant while holding } \mathrm{GO} / \mathrm{Fe}_{3} \mathrm{O}_{4} \text { composites } \\
\text { with a magnet; target analytes elution }(2 \times 1 \mathrm{~mL} \text { acetone containing } 1 \% \mathrm{HAc}) \text {; } \\
\text { evaporate to dryness }\left(60^{\circ} \mathrm{C} ; \mathrm{N}_{2} \text { stream); } \mathrm{MeOH}_{\text {reconstitution }(0.5 \mathrm{~mL}) \text {; filtration }}\right. \\
(0.45 \mu \mathrm{m}) ; \mathrm{HPLC} \text { analysis }(10 \mu \mathrm{L}) / 0.2-6.0 \mathrm{ng} \mathrm{mL}^{-1}\end{array}$ & $(60)$ \\
\hline MISM-PT & GA/juices & $\begin{array}{l}1 \mathrm{~mL} \mathrm{MeOH}+1 \mathrm{~mL} \text { water washing; } 6.0 \mathrm{~mL} \text { orange juice sample loaded on MISM-PT } \\
\left(0.15 \mathrm{~mL} \mathrm{~min}^{-1}\right) ; 150 \mu \mathrm{L} \text { hexane washing; } 250 \mu \mathrm{L} \text { gallic acid elution; extract } \\
\text { concentrated to dryness ( } \mathrm{N}_{2} \text { stream); dissolved in } 50 \mu \mathrm{L} \text { mobile phase; HPLC-UV } \\
\text { analysis }(20 \mu \mathrm{L}) / 7.0 \mu \mathrm{g} \mathrm{L}^{-1}\end{array}$ & $(61)$ \\
\hline PT-SPE & Estradiol/milk & $\begin{array}{l}\text { MIOMS-ir ( } 3 \mathrm{mg} \text { ) packed into } 100 \mu \mathrm{L} \text { pipette tip (cotton capped at both ends); } \\
\text { preconditioning ( } 1 \mathrm{~mL} \mathrm{MeOH}+0.5 \mathrm{~mL} \text { water); } 0.5 \mathrm{~mL} \text { milk sample loading; } 0.3 \mathrm{~mL} \\
\text { water washing; } 0.5 \mathrm{~mL} \mathrm{ACN} \text {-acetic acid }\left(96: 4, \mathrm{v} / \mathrm{v} \text { ) elution; evaporation to dryness }\left(\mathrm{N}_{2}\right.\right. \\
\text { by gentle nitrogen blowing); residue redissolving in the mobile phase }(0.5 \mathrm{~mL}) \text { for } \\
\text { LC-FLD analysis } / 6.00 \mathrm{ng} \mathrm{L}^{-1}\end{array}$ & $(62)$ \\
\hline SBA-15- $\mu$ SPE & Flavanones/citrus fruits & $\begin{array}{l}\text { Citrus edible parts blended; extract filtration; packed } 25 \mathrm{mg} \text { SBA-15 (1-mL cartridge); } \\
\text { preconditioning ( } 1 \mathrm{~mL} \mathrm{MeOH}, 1 \mathrm{~mL} \text { water); diluted juice }(20 \mu \mathrm{L}+4.97 \mathrm{~mL} \text { water, } \mathrm{pH} \\
\left.\text { 7) loading ( } 1 \mathrm{~mL} \mathrm{~min}^{-1} \text { flow rate); washing ( } 1 \mathrm{~mL} \text { water); vacuum elution ( } 1 \mathrm{~mL} \mathrm{MeOH}\right) \text {; } \\
\text { dryness (block heater); resuspension ( } 100 \mu \mathrm{L} \mathrm{MeOH);} \mathrm{UHPLC} \mathrm{analysis/4.26} \mathrm{ng} \mathrm{mL}^{-1}\end{array}$ & $(45)$ \\
\hline
\end{tabular}


TABLE 1 | Continued

\begin{tabular}{|c|c|c|c|}
\hline $\begin{array}{l}\text { Extraction } \\
\text { format/methodology }\end{array}$ & $\begin{array}{l}\text { Target } \\
\text { analytes/sample }\end{array}$ & Method description/analytical performance (LOD) & References \\
\hline SBA-15- $\mu$ SPE & $\begin{array}{l}\text { Triterpenoid } \\
\text { saponins/TCM }\end{array}$ & $\begin{array}{l}0.25 \mathrm{~g} \text { dried powder }+70 \% \text { EtOH ( } 25 \mathrm{~mL}) \text {; US (ice bath, } 30 \mathrm{~min}) \text {; filtration, dilution to } \\
50 \mathrm{~mL} \text {. SPE: packed } 25 \mathrm{mg} \text { SBA-15 (1-mL cartridge); preconditioning }(1 \mathrm{~mL} \mathrm{MeOH} \text {, } \\
1 \mathrm{~mL} \text { water); diluted extract loading ( } 1 \mathrm{~mL} \mathrm{~min}^{-1} \text { flow rate); washing }(1 \mathrm{~mL} \text { water); } \\
\text { vacuum elution ( } 1 \mathrm{~mL} \text { EtOH } 70 \%) ; \text { dryness }\left(\mathrm{N}_{2} \text { stream); resuspension }(200 \mu \mathrm{L}\right. \\
\text { MeOH); UHPLC-CAD analysis/0.461-0.976 } \mu \mathrm{g} \mathrm{mL}^{-1}\end{array}$ & (63) \\
\hline MEPS & $\begin{array}{l}\text { Furanic } \\
\text { derivatives/sugarcane } \\
\text { honey }\end{array}$ & $\begin{array}{l}\mathrm{eVo}^{\circledR} \text { syringe + RCX sorbent ( } 4 \text { mg); conditioning }(250 \mu \mathrm{L} \text { MEOH + } 250 \mu \mathrm{L} F A \\
0.1 \%) ; 500 \mu \mathrm{L} \text { sample loading (3 withdraw cycles); } 100 \mu \mathrm{L} \text { MeOH washing; } 100 \mu \mathrm{L} \\
\text { MeOH: FA 0.1\% (95:5, v/v) elution + } 100 \mu \mathrm{L} \text { FA 0.1\% elution; UHPLC-PDA } \\
\text { analysis/30.6-737.7 } \mu \mathrm{g} \mathrm{kg}^{-1}\end{array}$ & (64) \\
\hline$\mu$ SPEed & Phenolics/tea & $\begin{array}{l}\text { eVo }^{\circledR} \text { syringe }(200 \mu \mathrm{L} / \mathrm{min} \text { flow rate) }+ \text { PS/DVB-RP sorbent; conditioning }(500 \mu \mathrm{L} \\
\mathrm{MeOH}+200 \mu \mathrm{L} \text { FA 0.1\%); } 2 \times 100 \mu \mathrm{L} \text { sample loading; } 50 \mu \mathrm{L} \mathrm{MeOH} \text { : FA 0.1\% } \\
\text { (95:5, v/v) elution; UHPLC-PDA analysis }(2 \mu \mathrm{L}) / 3.5-16.8 \mathrm{ng} \mathrm{mL}^{-1}\end{array}$ & $(67)$ \\
\hline SE/dSPE & $\begin{array}{l}\text { Polyphenols/juice, } \\
\text { smoothies }\end{array}$ & $\begin{array}{l}50 \mathrm{mg} \text { of HMS-C18 conditioned with } 1 \mathrm{~mL} \mathrm{MeOH} / \text { water }(50: 50 \text {, v/v); } 10 \mathrm{~min} \text { stirring } \\
(300 \mathrm{rpm}) \text {; add } 5 \mathrm{~mL} \text { sample extract, } 20 \mathrm{~min} \text { stirring }(300 \mathrm{rpm}) \text {; filtration }(0.45 \mu \mathrm{m}) \text {; } \\
\text { elution }(2 \times 3 \mathrm{~mL} \mathrm{MeOH} / \text { water } 95: 5, \mathrm{v} / \mathrm{v} \mathrm{pH} 2) \text {; evaporation to dryness; } \mathrm{MeOH} \\
\text { resuspension }(500 \mu \mathrm{L}) \text {; UHPLC-MS analysis/0.01-1.7 } \mu \mathrm{g} \mathrm{mL}^{-1}\end{array}$ & (68) \\
\hline CNF-NLPNE & $\begin{array}{l}\text { Phytohormones, } \\
\text { ginsenosides/standard } \\
\text { solutions }\end{array}$ & 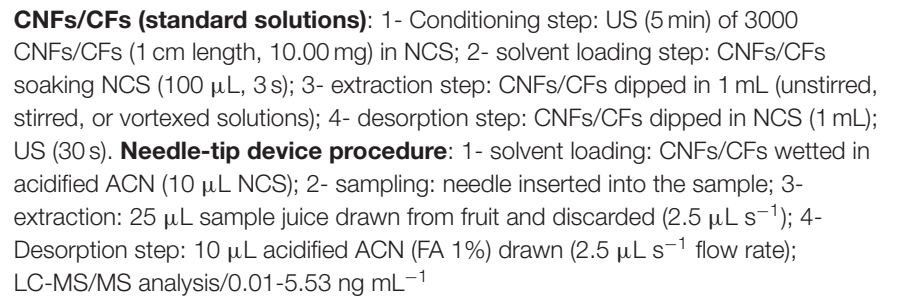 & (69) \\
\hline $\begin{array}{l}\text { M-DES-d } \mu S P E / M- \\
d \mu S P E\end{array}$ & $\begin{array}{l}\text { Morin, quercetin, } \\
\text { kaempferol/foods }\end{array}$ & 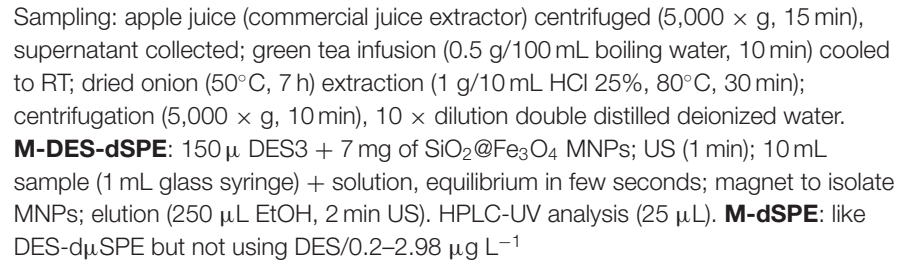 & $(48,70)$ \\
\hline MSPDM & Phenols/olive fruits & $\begin{array}{l}\text { Glass mortar blending }(60 \mathrm{~s}) \text { powdered sample }(50 \mathrm{mg})+25 \mathrm{mg} \\
\text { middle-molecular-weight chitosan (dispersant); column packing, plunger } \\
\text { compression; elution }(3 \times 0.5 \mathrm{~mL} \mathrm{MeOH}(60 \% \text {, v/v); evaporation to dryness; } \mathrm{MeOH} \\
\text { redissolution }(200 \mu \mathrm{L}) \text {; centrifugation ( } 5 \mathrm{~min}, 13,000 \mathrm{rpm}) \text {, UHPLC-Q-TOF/MS } \\
\text { analysis }(2 \mu \mathrm{L}) / 69.6-358 \mathrm{ng} \mathrm{g}^{-1}\end{array}$ & $(47)$ \\
\hline $\begin{array}{l}\text { Graphene } \\
\text { nanoplatelets- } \\
\text { MSPDM }\end{array}$ & $\begin{array}{l}\text { Phenolic acids/plant } \\
\text { preparations }\end{array}$ & $\begin{array}{l}\text { Graphene nanoplatelets and sample mixture (mortar) packed into 1-mL SPE } \\
\text { cartridge; } 0.2 \mathrm{~mL} \text { elution (different solvents); dilution ( } 10 \times \text {, elution solvent); } \\
\text { centrifugation ( } 5 \text { min, } 13,000 \text { rpm); UHPLC-ECD analysis ( } 2 \mu \mathrm{L}) / 1.19-4.62 \mathrm{ng} \mathrm{mL}^{-1}\end{array}$ & $(71)$ \\
\hline
\end{tabular}


TABLE 1 | Continued

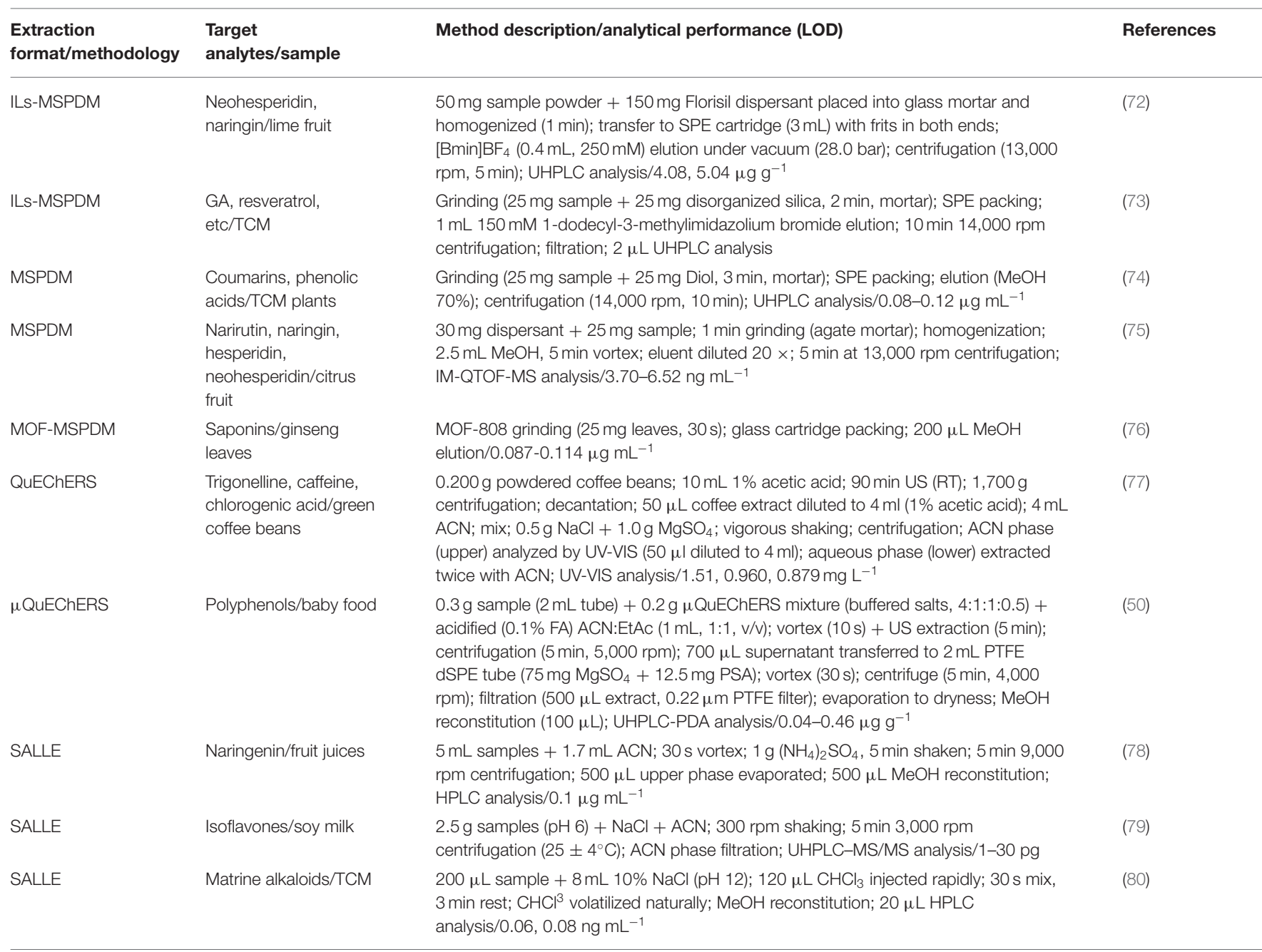

ACN, acetonitrile; BIN, barrel insert needle; [Bmin]BF 4, 1-butyl-3-methylimidazolium tetrafluoroborate; CNFs, carbon nanofibers; CNTS, carbon nanotubes; DES, deep eutectic solvents; dSPE, dispersive solid-phase extraction; DVB, divinylbenzene; ECD, electrochemical detection; EPT-SPME, effervescent pipette tip solid phase microextraction; EtOH, ethanol; FA,

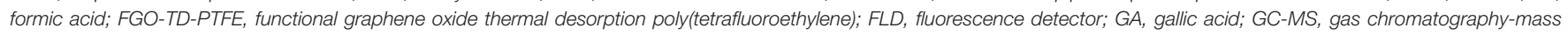

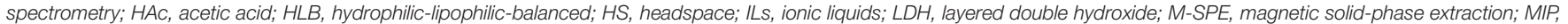

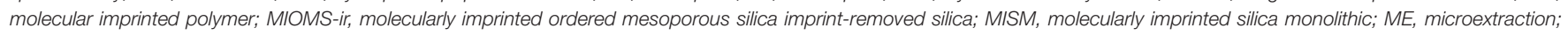

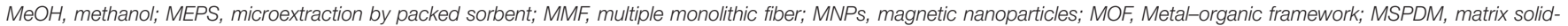

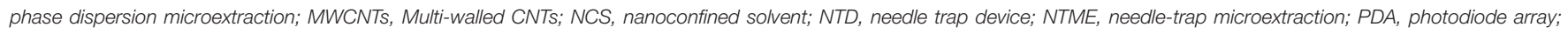

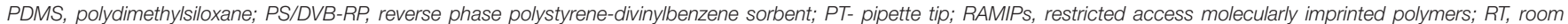

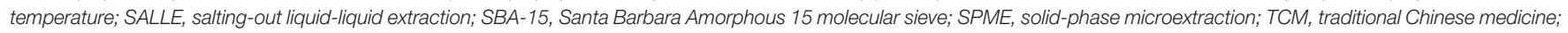
US, ultrasonication; VOCs, volatile organic compounds; UHPLC, ultrahigh performance liquid chromatography; UV, ultraviolet analysis; $\mu$ SPE, micro solid-phase extraction.

even lower submicron sorbent particles $(3 \mu \mathrm{m})$ and a controlled pressure-driven one-way valve that forces the sample through the sorbent particles in a single direction. Overall, these updates to MEPS configuration make $\mu$ SPEed even more powerful for a broad range of applications, preferentially involving samples with low viscosity to avoid clogging the sorbent containers (120). For this reason, most of the MEPS and $\mu$ SPEed applications reported so far involve liquid samples or solid samples that suffer a previous solvent extraction or dilution to decrease viscosity. Among the most recent and notable MEPS and $\mu$ SPEed applications reported in the literature in food analysis, we can find the extraction of phenolic acids in tea (67) and baby food (66) and furanic derivatives in sugarcane honey (Tables 1, 2).

\section{Microextraction Formats Involving the Dispersion of the Sorbent}

A popular approach to microextraction involves the blending of the sorbent with the sample matrix to facilitate the retention of the target analytes in the sorbent particles. This is the extraction principle in MSPD, dispersive SPE (dSPE) and related formats, which then diverge in the form as the sorbent particles and retained analytes are recovered. Often this is achieved by packing in columns, or simply in SPE tubes, to which vacuum is applied, or by centrifugation, followed by the target analytes elution in a suitable elution solvent. In this type of extractions applied to the extraction of bioactive compounds in food lies, the miniaturization to lower the different reagent 
TABLE 2 | Selected examples of microextraction of bioactive compounds in liquid sorbents.

\begin{tabular}{|c|c|c|c|}
\hline $\begin{array}{l}\text { Extraction } \\
\text { format/methodology }\end{array}$ & $\begin{array}{l}\text { Target } \\
\text { analytes/sample }\end{array}$ & Method description/LOD & References \\
\hline LLME & Caffeine/tea, coffee & $\begin{array}{l}20 \mathrm{mg} \text { tea leaves powder }+20 \mathrm{~mL} \mathrm{EtOH} / 10 \mathrm{~mL} \text { water (beaker, } \mathrm{pH} \text { adjusted to } 2.5 \text { ); } \\
\text { stirring }\left(40 \mathrm{~min}, 45^{\circ} \mathrm{C}\right) \text {; supernatant decantated and filtered }(0.45 \mu \mathrm{m}) ; 1.0 \mathrm{~mL} \\
\text { extracted sample in } 2: 1 \mathrm{EtOH} / \text { water mixture (conical glass test tube); } 150 \mu \mathrm{L} \mathrm{DCM} \\
\text { rapidly injected to form three solvents mixture; } 20 \text { s high speed vortex; add } 300 \mu \mathrm{L} \\
\text { water; vigorous shake, rest to obtain two phases; caffeine sedimented (bottom } \\
\text { organic phase of DCM); centrifugation ( } 5,000 \mathrm{rpm}, 4 \mathrm{~min}) / 0.05 \mu \mathrm{g} \mathrm{mL}^{-1}\end{array}$ & (81) \\
\hline VA-LLME & $\begin{array}{l}\text { Phenolic acids/honey, } \\
\text { iced tea, coffee drinks }\end{array}$ & $\begin{array}{l}\text { Sample solution ( } \mathrm{pH} 1.5-1.8,10 \mathrm{~mL} \text { volumetric flask); propyl acetate: pentanol: } \\
\text { hexanol (1:2:1.5, v/v/v, } 400 \mu \mathrm{L}) \text { vortex extraction }(45 \mathrm{~s}, 2,500 \mathrm{rpm}) ; \text { rest } 1 \mathrm{~min} \text {; } \\
\text { transfer top layer ( } 200 \mu \mathrm{L}) \text { to centrifuge tube }(0.5 \mathrm{~mL}) \text { containing } 40 \mu \mathrm{L} \mathrm{KOH} \\
(0.02 \mathrm{M}) \text {; vortex }(60 \mathrm{~s}, 2,500 \mathrm{rpm}) \text {; collect aqueous fraction (bottom layer); HPLC } \\
\text { analysis/0.05-0.68 } \mu \mathrm{g} \mathrm{L}^{-1}\end{array}$ & $(82)$ \\
\hline VA-LLME & $\begin{array}{l}\text { Hydrophilic } \\
\text { phenols/olive oil }\end{array}$ & $\begin{array}{l}5 \mathrm{~mL} \text { oil samples diluted to } 5 \mathrm{~mL} \text { (hexane) }+100 \mu \mathrm{L} 1 \mathrm{M} \mathrm{HCl} ; 2 \text { min vortex; } 10 \mathrm{~min} \\
\text { 4,000 rpm centrifugation; } 40 \mu \mathrm{L} \text { acidic aqueous phase (lower phase) analyzed by } \\
\text { differential pulse voltammetry (DPV) using SPCEs/0.022 } \mathrm{mg} \mathrm{L}^{-1}\end{array}$ & (83) \\
\hline $\begin{array}{l}\text { SHS- } \\
\text { LLME/DLLME }\end{array}$ & Piperine/pepper & $\begin{array}{l}\text { Sample grinding; } 0.10 \mathrm{~g}+5.0 \mathrm{ml} \text { ACN } 45 \% \text { (v/v); } 1 \text { min vortex; } 2 \text { min } 6,000 \mathrm{rpm} \text {; } \\
\text { filtration; } 3.0 \mathrm{~mL} \text { saturated } \mathrm{NaCl} \text { (Salting-out extraction }-\mathrm{SOE} \text { ); } 1 \mathrm{~min} \text { vortex; } 2 \mathrm{~min} \\
6,000 \mathrm{rpm} ; 50 \mu \mathrm{L} \text { SOE diluted to } 4.0 \mathrm{~mL} \text { (deionized water); } 1.5 \mathrm{~mL} \text { SHS }+1.0 \mathrm{~mL} \\
20 \mathrm{M} \mathrm{NaOH}, 10 \mathrm{~s} \text { vortex; recover switched-off SHS layer; dilute } 50 \% \text { prior HPLC-DAD } \\
\text { analysis/0.2-0.6, } 0.7-2.0 \mu \mathrm{g} \mathrm{mg}^{-1}\end{array}$ & (84) \\
\hline $\begin{array}{l}\text { LLME (US and } \\
\text { salt-assisted) }\end{array}$ & Oleuropein/olive oil & $\begin{array}{l}0.01 \mathrm{~g}+10 \mathrm{~mL} \text { extraction solvents mixture (phosphate buffer, with variable } \mathrm{pH}, \mathrm{ACN} \text {, } \\
\left.\text { and THF); US ( } 25^{\circ} \mathrm{C}\right) ; 5 \mathrm{~min} 4,000 \mathrm{rpm} \text { centrifugation; } 1 \mathrm{~mL} \text { liquid phase }+\mathrm{NaCl} \\
\text { (salting out); } 10 \mu \mathrm{L} \text { organic phase HPLC analysis } / 0.5 \mu \mathrm{gL}^{-1}\end{array}$ & (85) \\
\hline $\begin{array}{l}\text { Ball mill-assisted } \\
\text { DES-based } \\
\text { extraction }\end{array}$ & $\begin{array}{l}\text { Tanshinones/Salvia miltiorrhiza } \\
\text { Bunge }\end{array}$ & $\begin{array}{l}\text { Oven-dried, sliced, and crushed samples }(0.05 \mathrm{~g}) \text { mixed with DES }(1.0 \mathrm{~mL}) \text { in } 2.0 \mathrm{~mL} \\
\text { Lysing Matrix D tubes }(1.4 \mathrm{~mm} \text { ceramic spheres, } 1.1 \mathrm{~g}) \text {; centrifugation; LC-MS } \\
\text { analysis }\end{array}$ & (86) \\
\hline DES-LLME & Caffeine/soft drinks & $\begin{array}{l}1 \mathrm{~mL} \text { sample; } 50 \mu \mathrm{L} \text { THF (aprotic solvent); } 50 \mu \mathrm{L} \text { DES (choline chloride-phenol); } \\
\text { HPLC-UV analysis/0.03 } \mu \mathrm{g} \mathrm{mL}^{-1}\end{array}$ & $(87)$ \\
\hline DES-LLME & Quercetin/wine, foods & $\begin{array}{l}\text { Ground dried }(5 \mathrm{~g}) \text { and liquid }(3 \mathrm{~mL}) \text { samples }+10 \mathrm{~mL} \mathrm{MeOH} ; 40 \text { min US extraction } \\
(\mathrm{RT}) \text {; filtration } / 6.1 \mathrm{~g} \mathrm{~L}^{-1}\end{array}$ & (88) \\
\hline DES-LLME & $\begin{array}{l}\text { Rare } \\
\text { ginsenosides/Kang'ai } \\
\text { injection }\end{array}$ & $\begin{array}{l}8 \mathrm{~mL} \text { sample }+400 \mu \mathrm{L} \text { DES in glass test tube; add } 150 \mathrm{mg} \text { inorganic salt; shake to } \\
\text { obtain homogeneous solution; add } 400 \mu \mathrm{L} \mathrm{THF} \text { (turbidity is observed); add } 150 \mathrm{mg} \\
\mathrm{Fe}_{3} \mathrm{O}_{4} \text {; inject } \mathrm{N}_{2} \text { (to obtain homogenous turbid droplets and make the magnetic } \\
\text { nanoparticles absorb the droplets simultaneously); collect nanoparticles (magnet); } \\
\text { washed thoroughly (little amount EtOH); concentrate to } 200 \mu \mathrm{L} \text {; filtration ( } 0.22 \mu \mathrm{m} \\
\text { PTFE); HPLC analysis/10.2-137.8 } \mathrm{ng} \mathrm{mL}{ }^{-1}\end{array}$ & (89) \\
\hline DES-LLME & $\begin{array}{l}\text { Curcumin/food, herbal } \\
\text { tea }\end{array}$ & $\begin{array}{l}\text { Curcumin standards + phosphate buffer ( } 2 \mathrm{~mL}, \mathrm{pH} 4) \text { in } 50 \mathrm{~mL} \text { centrifuge tube; } 400 \\
\mu \mathrm{L} \text { DES (ChCl: Phenol, 1:4) as water-miscible extraction solvent injected rapidly into } \\
\text { solution to form homogeneous solution; THF ( } 400 \mu \mathrm{L} \text {, emulsifier) injected into } \\
\text { solution; cloudy solution (formation of insoluble self-aggregates) was obtained; US } \\
\text { ( } 2 \text { min) to homogenize DES droplets in the aqueous phase (curcumin extraction); } \\
\text { centrifugation ( } 4,500 \mathrm{rpm}, 5 \mathrm{~min}) \text {; discard top water phase; DES rich-phase } \\
\text { containing curcumin completed to } 1 \mathrm{~mL} \text { with EtOH; curcumin concentration } \\
\text { determined by UV }(425 \mathrm{~nm}) / 2.86 \mu \mathrm{g} \mathrm{L}^{-1}\end{array}$ & (90) \\
\hline DES-DLLME & $\begin{array}{l}\text { Phenylpropanoids/vegetable } \\
\text { oils }\end{array}$ & $\begin{array}{l}2 \mathrm{~mL} \text { oil samples }+2 \mathrm{~mL} n \text {-hexane; } 45 \mu \mathrm{L} \text { PhAA/TMG in } 200 \mu \mathrm{L} \text { isopropanol } \\
\text { (dispersing solvent) rapidly added; } 10 \mathrm{~s} \text { vortex; } 5,000 \mathrm{~g} \text { centrifugation ( } 5 \mathrm{~min} \text { ); } 5 \mu \mathrm{L} \\
\text { DES solution UHPLC analysis/0.07-0.01 } \mu \mathrm{g} \mathrm{mL}^{-1}\end{array}$ & $(91)$ \\
\hline $\begin{array}{l}\text { DLLME (OS vs. } \\
\text { DES) }\end{array}$ & Phytosterols/cow milk & $\begin{array}{l}2.0 \mathrm{~mL} \text { milk sample; } 1.0 \text { or } 1.25 \mathrm{~mL} \text { ACN (OS-DLLME or DES-DLLME, respectively); } \\
30 \text { s vortex; } 4 \mathrm{~min} 4,000 \mathrm{rpm} \text { centrifugation; supernatant phase }(0.8 \mathrm{~mL})+70 \mu \mathrm{L} \\
\mathrm{CCl}_{4} \text {, mix; } 3 \mathrm{~min} 4,000 \mathrm{rpm} \text { centrifugation; bottom phase evaporated }\left(\mathrm{N}_{2} \text { stream); }\right. \\
\mathrm{ACN}(95 \%, \mathrm{v} / \mathrm{v}) \text { reconstitution; HPLC-UV analysis/0.3-0.9, 0.09-0.32 } \mathrm{ng} \mathrm{mL}^{-1}\end{array}$ & (92) \\
\hline NADES-LLME & $\begin{array}{l}\text { Polyphenols/Greek } \\
\text { medicinal plants }\end{array}$ & $\begin{array}{l}\text { Pulverized material }(0.1 \mathrm{~g})+\mathrm{NADES}(80 \% \mathrm{v} / \mathrm{v}, 10 \mathrm{~mL}) \text {, manual vigorous shaking; US } \\
\left.\text { extraction }\left(80^{\circ} \mathrm{C}, 90 \mathrm{~min}, 140 \mathrm{w}\right) \text {; centrifugation ( } 15,000 \mathrm{rpm}, 10 \mathrm{~min}\right) \text {; dilution 1:20 } \\
\text { distilled water; spectrometric analysis }\end{array}$ & (93) \\
\hline NADES-LLME & $\begin{array}{l}\text { Anthocyanins/Catharanthus } \\
\text { roseus }\end{array}$ & $\begin{array}{l}\text { Samples }(50 \mathrm{mg})+\text { NADES }(1.5 \mathrm{ml}) \text {; stirred }\left(40^{\circ} \mathrm{C}, 30 \mathrm{~min}\right) \text {; centrifugation }(1,300 \mathrm{rpm} \text {, } \\
20 \mathrm{~min}) \text {, filtration }(0.45 \mu \mathrm{m} \text { filter); dilution }(1: 2,3 \% \mathrm{FA}) \text {; HPLC-DAD analysis }\end{array}$ & (94) \\
\hline
\end{tabular}


TABLE 2 | Continued

\begin{tabular}{lll}
\hline $\begin{array}{l}\text { Extraction } \\
\text { format/methodology }\end{array}$ & $\begin{array}{l}\text { Target } \\
\text { analytes/sample }\end{array} \quad$ Method description/LOD
\end{tabular}

\section{format/methodology analytes/sample}

DLLME/SULLE Phenolics/plums (Prunus domestica L.)

DLLME (several variations)/SULLE

DLLME

DLLME

Tocopherol/bovine milk

$\begin{array}{ll}\text { DLLME } & \begin{array}{l}\text { Flavonols, } \\ \text { organosulfurs, } \\ \text { inulin/garlic, foods } \\ \text { DLLlatonin and } \\ \text { trans-resveratrol/Wine }\end{array} \\ & \\ \text { In-syringe DLLME } & \text { Caffeine/coffee } \\ \text { DLLME } & \begin{array}{l}\text { Caffeine/tea; energy } \\ \text { drinks }\end{array}\end{array}$

DES-HS-SDME Terpenes/spices

M-ILs-SDME Ascorbic acid (AA)/orange juice
DLLME: $10 \mathrm{mg}$ dry leaves into Eppendorf tube $+1 \mathrm{~mL}$ extraction medium; $200 \mu \mathrm{L}$ EtAc + $100 \mu \mathrm{L}$ ACN fast injection; cloudy solution centrifugation; injection (30 s); rest 1 min, 5 min $12,000 \times g$ centrifugation; $100 \mu \mathrm{L}$ top layer evaporated $\left(\mathrm{N}_{2}\right.$ stream); 50 $\mu \mathrm{L}$ mobile phase resuspension, US (5 min); $20 \mu \mathrm{L}$ HPLC analysis. SULLE: $10 \mathrm{mg}$ dry leaves $+200 \mu \mathrm{L}$ water $+400 \mu \mathrm{L} \mathrm{ACN}$; gentle shaking (30 s); $200 \mu \mathrm{L}$ sugar solution rapidly injected, 1 min vortex; phase separation centrifugation (5 min 12,000 × g); 250 $\mu \mathrm{L}$ top layer dried ( $\mathrm{N}_{2}$ stream); $50 \mu \mathrm{L}$ mobile phase resuspension, US (5 min); $20 \mu \mathrm{L}$ HPLC analysis.

$50 \mathrm{mg}$ ground roots (40 mesh) $+5 \mathrm{~mL}$ water (or $10 \% \mathrm{NaCl}$, NADES, IL, glucose or $1 \% \beta$-cyclodextrin and HP- $\beta$-cyclodextrin); 30 s gentle shaking, $600 \mu \mathrm{L}$ EtAc (extraction solvent) $+500 \mu \mathrm{l}$ ACN (dispersive solvent); 30 s vortex, 2 min rest (10 min US for UA-DLLME); 4 min 1,500 rpm centrifugation; recover $350 \mu \mathrm{L}$ top layer; dry $\left(\mathrm{N}_{2}\right.$ stream); $50 \mu \mathrm{L}$ resuspension (7\% ACN, v/v, 3\% acetic acid), $20 \mu \mathrm{L}$ HPLC analysis.

Sample $(2 \mathrm{~mL})+4 \mathrm{~mL}$ water $(5 \mathrm{~mL}$ glass centrifuge tube); fast mixture injection - 250 $\mu \mathrm{L} \mathrm{EtOH} \mathrm{(dispersant)}+70 \mu \mathrm{L}$ bromocyclohexane (extractant); vortex (10 s), US (2 min, $40 \mathrm{KHz}$ ); centrifugation (2.5 min, 13,457 $\times \mathrm{g}$ ); sedimented phase transferred to another vial, dry ( $\mathrm{N}_{2}$ stream); add $120 \mu \mathrm{L}$ CSR + $50 \mu \mathrm{L} C M P I+50 \mu \mathrm{L}$ DMAP ACN; sealed vial radiated $\left(750 \mathrm{~W}\right.$ microwave reactor, $\left.5 \mathrm{~min}, 60^{\circ} \mathrm{C}\right)$; dilute resulting solution with $4.0 \mathrm{~mL}$ water ( $5 \mathrm{~mL}$ centrifuge tube); add $70 \mu \mathrm{L}$ bromobenzene (extractant) + $220 \mu \mathrm{L}$ ACN (dispersant); vortexed (20 s), US (2 min); centrifugation (2.5 min, 13,457 $\times$ g); recover bromobenzene (bottom phase); UHPLC-MS/MS analysis/0.005-0.015 $\mathrm{ng} \mathrm{mL} \mathrm{m}^{-1}$

$1.0 \mathrm{~mL}$ milk sample $+9.0 \mathrm{~mL} \mathrm{EtOH}$ (containing ascorbic acid, $5 \mathrm{~g} / \mathrm{L}$ ) heating $\left(78^{\circ} \mathrm{C}\right.$, $30 \mathrm{~min}, 10 \mathrm{~min}$ intervals shake); ice-cooling, centrifugation ( $5 \mathrm{~min}, 4,500 \mathrm{rpm}$ ); $1.0 \mathrm{~mL}$ supernatant $+200 \mu \mathrm{L}$ chloroform rapidly injected into $5 \mathrm{~mL}$ ultrapure water; cloudy solution centrifuged (5 min, 4,500 rpm); organic phase centrifuged again (10 min, $13,500 \mathrm{rpm})$, HPLC-PDA analysis/0.01 $\mu \mathrm{g} \mathrm{mL}^{-1}$

$600 \mu \mathrm{L}$ chloroform (extraction solvent) $+1 \mathrm{~mL}$ ACN (dispersive solvent) injected into sample solution; centrifugation (3 min, 2,000 rpm); chloroform phase dried $\left(\mathrm{N}_{2}\right.$ stream); $\mathrm{MeOH}$ reconstitution (500 $\mu \mathrm{L}) ; \mathrm{HPLC}-\mathrm{DAD}$ analysis/0.14-2.15 $\mu \mathrm{g} \mathrm{mL}^{-1}$

$2 \mathrm{~mL}$ centrifuged sample $+7 \mathrm{~mL}$ ultrapure water; $1.500 \mu \mathrm{L} \mathrm{ACN}$ (disperser) +300 $\mu \mathrm{L}$ chloroform (extracting solvent) $+1.500 \mathrm{mg} \mathrm{NaCl}$ (ionic strength); mix $1 \mathrm{~min} ; 5 \mathrm{~min}$ centrifugation; evaporate the organic phase $\left(\mathrm{N}_{2}\right.$ steam); re-dissolution (150 $\mu \mathrm{L}$ phosphate buffer (40 mM), pH 3/ACN (80/20, v/v); HPLC-FLD analysis/0.07, 7.68 ng $\mathrm{mL}^{-1}$

1,500 $\mu \mathrm{L}$ sample $+(1,225 \mu \mathrm{L} \mathrm{MeOH}+225 \mu \mathrm{L}$ DCM extraction); 20 s stirring, repeat; extract water dilution, filtration, HPLC-UV analysis $/ 0.46 \mu \mathrm{g} \mathrm{mL}^{-1}$

$2 \mathrm{~mL}$ sample $+8 \mathrm{~mL}$ deionized water; $1 \mathrm{~min}$ shaking; $\mathrm{pH}$ adjusted to $3 ;+1.5 \mathrm{~g} \mathrm{NaCl}$; mix vigorously; + $450 \mu \mathrm{L} \mathrm{EtOH} \mathrm{(disperser)}+80 \mu \mathrm{L}$ 1-octanol (extraction solvent); 1 min mixing; 6,000 rpm (5 min) centrifugation; $20 \mu \mathrm{L}$ upper phase HPLC-UV analysis/0.9 $\mathrm{ng} \mathrm{mL}^{-1}$

50 mg sample ( $20 \mathrm{~mL}$ HS vial); $10 \mu \mathrm{L}-\mathrm{GC}$ microsyringe containing DES introduced in the HS of the sample vial: DES pushed down the microsyringe to form $1.5 \mu \mathrm{L}$ drop at the needle tip; incubation $\left(80^{\circ} \mathrm{C}, 90 \mathrm{~min}\right)$; DES drop withdrawn into the microsyringe, disposed in $250 \mu \mathrm{L}$ insert and weighed; GC-MS analysis/0.47-86.40 $\mu \mathrm{g} \mathrm{g}^{-1}$

$8 \mathrm{mg} \mathrm{M}$-ILs dissolved in a single $\mathrm{EtOH}$ droplet (1.0 $\mu \mathrm{L}$ ); place on surface of $2 \mathrm{~mL}$ phosphate buffer $(0.10 \mathrm{M}$, pH 6.0) containing AA (1.50- $40.0 \mathrm{nM})$; mixture gently stirred (15 min); AA extracted into M-ILs phase small volume; M-ILs-rich phase collected (strong magnet out the wall of the solution-containing tube, supernatant decanted). M-ILs phase EtOH dilution (3 $\mu \mathrm{L}$ ); transfer onto the TiO2-NPs/CPE surface; $2 \mu \mathrm{L}$ Nafion casted on the electrode; solvent evaporation (RT)/0.43 nM $1.0 \mathrm{~g}$ licorice powder (pulverized, 30 mesh sieves) $+10 \mathrm{~mL}$ ILs (several mixtures); US extraction; centrifugation; filtration; HPLC analysis/0.002-0.067 $\mu \mathrm{g} \mathrm{mL}^{-1}$

79.6 mg saffron sample; $1.1 \mathrm{~mL} \mathrm{H}_{2} \mathrm{O}$ (extraction solvent); 18.6 min sonication $62.7 \mu \mathrm{L}$ chloroform (pre-concentration solvent); RP-HPLC-DAD analysis
(95) 
TABLE 2 | Continued

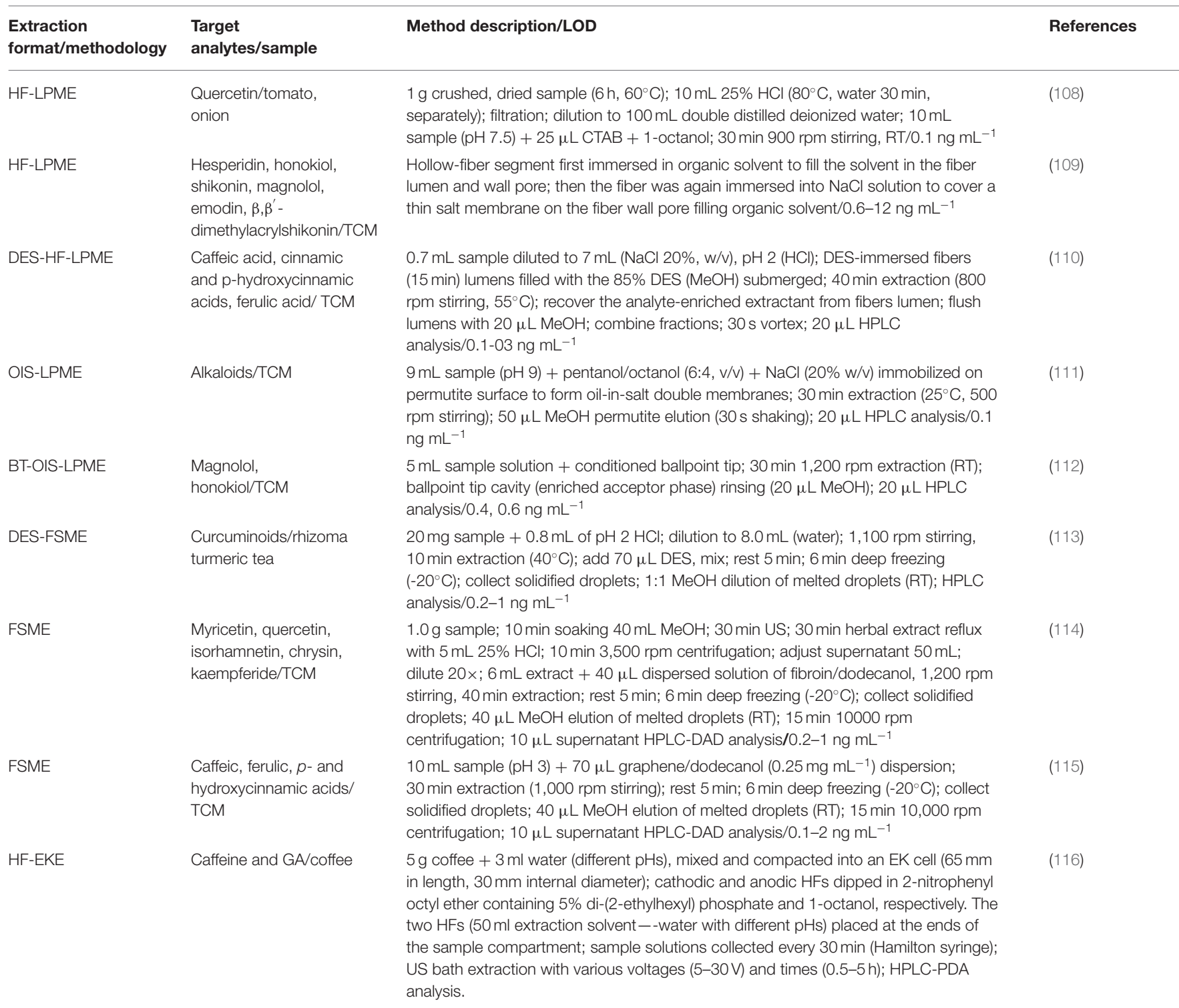

AA, Ascorbic acid; ACN, acetonitrile; APCI-MS, atmospheric-pressure chemical ionization mass spectrometry; BT-IOS-LPME, Ballpoint tip-protected oil-in-salt liquid-phase microextraction; CMPI, 2-Chloro-1-Methyl Pyridinium lodide; CSR, 4'-Carboxy-substituted rosamine; DAD, diode-array detection; DCM, dichloromethane; DES, deep eutectic solvents; DLLME- dispersive liquid-liquid microextraction; DMAP, 4-dimethylaminopyridine; EtAc, ethyl acetate; EtOH, ethanol; FA, formic acid; FSME, floating solidification microextraction; GA, gallic acid; GC-MS, gas chromatography-mass spectrometry; HF-EKE, hollow fiber electrokinetic extraction; HF-LPME, hollow-fiber liquid-phase microextraction; HS, headspace; ILs, ionic liquids; IOS-LPME, oil-in-salt liquid-phase microextraction; LC-MS, liquid chromatography mass spectrometry; LE, liquid extraction; LLME, liquid-liquid microextraction; M-SPE, magnetic solid-phase extraction; M-ILs-SDME, magnetic ionic liquids single drop microextraction; ME, microextraction; MeOH, methanol; NADES, natural deep eutectic solvents; OS, organic solvents; PLE, pressurized liquid extraction; SHS, Switchable-hydrophilicity solvent; SULLE, sugaring-out assisted liquid-liquid extraction; TCM, traditional Chinese medicine; THF, Tetrahydrofuran; US, ultrasonication; USAEME, ultrasonic assisted emulsification microextraction; VA, vortex assisted.

and particles requirements seems inevitable, as reported to the extractions of phenolics from plant extracts (72) and juices (68). The use of magnetic nanoparticles in the extraction process constitute another important improvement because a simple magnet can be used to isolate the sorbent particles and retain analytes by decantation. This approach has been used to extract different flavonoids from tea and wines (60) and it is being incorporated in other formats to simplify the experimental design of the extraction process, but also the efficiency and selectivity of the methodology. This was achieved, for instance, by coupling magnetic particles with molecular imprinting, as reported in the extraction of different phenolic compounds from fruits and juices $(58,59)$. But eventually, the major innovation in microextraction of bioactive compounds in food analysis is the use of new and more efficient sorbents, allowing greater retention capacities, as a $2 \mathrm{D}$-ultrathin $\mathrm{Ni} / \mathrm{Co}-$ $\mathrm{NO}_{3}$ layered double hydroxide nanosheet (46) or graphene nanoplatelets (71) to extract phenolic acids from fruit juices 
and plant preparations. Also, carbon nanofibers (CNFs) were reported to extract phytohormones and ginsenosides (69).

\section{Microextraction Formats Using Liquid Sorbents}

In simple terms, conventional LLE involves the transference (extraction) of analytes present in a liquid sample to an organic and immiscible solvent to which the analytes have more affinity. This usually involves agitation (by mixing, vortexing, etc.) to facilitate the extraction, followed by centrifugation to separate and recover the phase containing the extracted analytes. Often, LLE uses large volumes of organic solvents and consequently generate a large volume of toxic wastes difficult to manage. The miniaturization of LLE contributes to mitigating these problems and several improvements at this level have been proposed. Amini and Hashemi (81), for instance, proposed an LLME approach to extract caffeine from tea and coffee using considerably fewer volumes of organic solvents than conventional LLE. In turn, Shalash et al. (82) reported an LLME using vortex to facilitate the extraction of phenolics from honey, ice tea, and coffee drinks. In the dispersive LLE (DLLE), the organic solvent usually forms micelles in the liquid phase containing the target analytes that increase considerably the extraction efficiency and similarly to LLME, several DLLME approaches have been reported in the literature to extract bioactive compounds from foods, like phenolics (95), vitamin E (98), phytosterols (97) or flavonols, organosulfurs and inulin (99, 100). Related to DLLME, single-drop microextraction (SDME) seems to be also a very efficient extraction procedure, allowing a great preconcentration factor as the volume of the extraction solvent is limited to a single drop. In the original instrumental configuration of SDME, the drop of the extracting (immiscible) solvent is controlled by a microsyringe (or pipette) that loads and withdraw the drop from the solution. This format is not easy to automatize and is often performed with non-commercial devices. However, Jahromi et al. (105) proposed an SDME using magnetic ILs (M-ILs), dispersing the single drop of the solvent containing the M-ILs in the sample solution and recovering it back using a magnet. Using this approach, the authors reported a methodology with a LOD of $0.43 \mathrm{nM}$ for the analysis of AA in orange juice. Liquid-phase microextraction is another interesting approach involving microextraction in liquid phases. The HFLPME is eventually the most popular approach of this type of microextraction, but recent reports propose other type of containers in which the microextraction of the target analytes occurs, as well as the use of DES. Gao et al. (111), for instance, immobilized the extraction solvent mixtures on a permutite surface to form oil-in-salt double membranes to extract active alkaloids from a TCM powder. In turn, Wang et al. (112) used a protected ballpoint tip to perform a similar oil-in-salt microextraction (IOS-LPME) of the phenolic lignans magnolol and honokiol, that constitute the main primary active ingredients of a TCM. Finally, Zhang et al. (110) reported deep eutectic solvent-based HF-LPME to extract cinnamic acid derivatives in TCM. Floating solidification microextraction (FSME) is an elegant variation of LPME involving the solidification of the extracting phase. Upon the extraction procedure, the enriched phase containing the extracted analytes is fast frozen and easily separated from solution by decantation. Then it is melted at RT and the target analytes re-extracted with $\mathrm{MeOH}$ and recovered by centrifugation. This approach has been successfully used to extract important bioactive compounds from different food matrices (Table 2).

\section{Microextraction Approaches Using Ionic Liquids}

The use of ILs in microextraction holds great potential due to their unique "green" properties including thermal stability, re-usage, high reaction efficiency and selectivity at room temperature and ability to dissolve both organic and inorganic compounds (121). For these reasons, ILs are being used in a growing number of applications, like the one referred above using magnetic ILs to extract AA (105). But the future of ILs belongs to the DES, a less toxic and even more ecofriendly subclass of ILs that are easier and cheaper to obtain $(122,123)$. DES provide a network of hydrogen bond acceptor (HBA) and hydrogen bond donor (HBD) that favor the dissolution process of target analytes (122) and their utility in the microextraction process is not restricted to their use as extraction solvent. DES are also useful to modify and improve the extraction efficiency and selectivity of other sorbents like nanoparticles and silica; to facilitate the dissolution or digestion of solid samples; and to act as elution solvent $(123,124)$. On top of this, there are natural occurring DES (NADES), that are even more interesting and promising in microextraction, particularly as substitutes of the conventional organic solvents. Bakirtzi et al. (93), for instance, assayed several NADES and showed that they were non-toxic, renewable, and exceptionally efficient solvents for polyphenol recovery from medicinal plants. This higher efficiency is very important because there are many compounds present in plant composition, that despite their important bioactivities, are present in so low concentrations that they remain unidentified (125). Phenolics are certainly one of such family of compounds and for this most of the reports in the literature using DES and different microextraction approaches applied to food matrices have phenolic compounds as target analytes $(86,89,90,93,94$, $104,125)$. In this context, the work reported by Majidi and Hadjmohammadi $(48,70)$ combining magnetic nanoparticles and DES in a dSPE format to extract morin, quercetin and kaempferol from different foods is particularly inspiring for other demanding applications envisaging target analytes in very low concentrations in the respective samples (Table 1). Further details of the experimental conditions used in this, and other reports involving DES are available in Tables 1, 2.

\section{Other Microextraction Approaches}

In this section were included microextraction formats involving different mechanisms of retention of target analytes. This is the case of the salting-out mechanism behind the popular $\mathrm{QuEChERS} \mathrm{extraction.} \mathrm{In} \mathrm{principle,} \mathrm{this} \mathrm{would} \mathrm{not} \mathrm{be} \mathrm{considered}$ a microextraction approach considering the different salt and solvent volumes required. However its miniaturization, $\mu \mathrm{QuEChERS}$, only requiring a fraction of the original recipe of 
QuEChERS, has been successfully optimized for the extraction and simultaneous determination by UHPL-UV analysis of 12 polyphenols in different baby food samples (50). Similarly, it is relevant to point recent salting-out liquid-liquid extraction (SALLE) approaches able achieve great improvements in the extraction and subsequent analysis of important bioactive compounds. This is the notable examples of the SALLE approach proposed by Park and Jung (79) to extract isoflavones from soy milk, which is 100 times faster than the conventional methodologies used with the same objective. Additional examples to extract naringenin from juices (78) or matrine alkaloids from TCM (80) are described in more detail in Table 1. The use of electric potential in sample microextraction is another format that has been reported for the analysis of bioactive compounds in food. Khajeh et al. (116) proposed a hollow fiber-electrokinetic extraction approach (HF-EKE) for the simultaneous extraction of GA and caffeine from coffee. This is a simple and promising approach but still requires further improvement to lower the extraction times to acceptable values ( $50 \mathrm{~h}$ to achieve $95 \%$ extraction, only $20 \%$ target analytes extraction after $5 \mathrm{~h}$ ).

\section{FINAL REMARKS AND OUTLOOK FOR FUTURE FORMATS AND APPLICATIONS}

In this review, the most recent and innovative microextraction approaches used to extract bioactive compounds from food samples have been presented and their potential and contribution to the whole analytical layout discussed. The major challenges many innovative microextraction approaches must face include their broad application in terms of suitable samples and target analytes, but mainly in terms of potential for their production and distribution across the scientific community. Beyond the analytical aspects, a successful microextraction application needs to be consistently produced and distributed at a commercial level, allowing its usage across different labs and comparison of the results obtained. In this context, the adoption of DES to substitute many conventional organic solvents holds great potential because can be readily used with the sampling and microextraction formats available, not requiring the adoption of new methodologies or acquisition of laboratory materials or instruments and will certainly improve the analytical performance of the currently used methodologies. In contrast, microextraction approaches

\section{REFERENCES}

1. Zhou Y, Zheng J, Li Y, Xu DP, Li S, Chen YM, et al. Natural polyphenols for prevention and treatment of cancer. Nutrients. (2016) 8:0515. doi: 10.3390/nu8080515

2. Sharma A, Kaur M, Katnoria JK, Nagpal AK. Polyphenols in food: cancer prevention and apoptosis induction. Curr Med Chem. (2018) 25:474057. doi: 10.2174/0929867324666171006144208

3. Poti F, Santi D, Spaggiari G, Zimetti F, Zanotti I. Polyphenol health effects on cardiovascular and neurodegenerative disorders: a review and meta-analysis. Int J Mol Sci. (2019) 20:351. doi: 10.3390/ijms20020351 involving custom sorbents, like MIPS, CNTs and CNFs, despite the superior analytical performance they may allow, will be adopted only if they become commercially available. In turn, MNPs still require additional studies involving their regeneration and durability without affecting the performance of the extraction process. Beyond the type and sorbents feasibility, the microextraction format is also an important aspect to consider, namely the simplicity of the operation mode and the possibility for and easier migration from manual to automatic modes. In this point, miniaturized SPE formats, like $\mu$ SPEed, will continue to benefit from using traditional formats, but their potential can certainly be further enhanced with the adoption of even more efficient sorbent particles. Eventually, the adoption of DES in the $\mu$ SPEs formats will make microextraction even more efficient, boosting analytical performance to fulfill the challenges that food analysis involves.

\section{AUTHOR CONTRIBUTIONS}

JP, NC, and JC contributed to the planning of the review. JP, NC, PP-F, and JC wrote sections of the manuscript. JP edited the manuscript. All authors contributed to manuscript revision, read, and approved the submitted version.

\section{FUNDING}

This work was supported by FCT-Fundação para a Ciência e a Tecnologia through the CQM Base Fund - UIDB/00674/2020, and Programmatic Fund - UIDP/00674/2020, and by ARDITIAgência Regional para o Desenvolvimento da Investigação Tecnologia e Inovação, through the project M1420-01-0145FEDER-000005 - Centro de Química da Madeira - CQM+ (Madeira 14-20 Program). JP was supported by a Post-Doctoral fellowship given by ARDITI (Project M1420 - 09-5369-FSE000001) and Priscilla Porto-Figueira was supported by FCT (Ph.D. fellowship SFRH/BD/129630/2017).

\section{ACKNOWLEDGMENTS}

The authors acknowledge the financial support from Fundação para a Ciência e Tecnologia and Madeira 14-2020 program to the Portuguese Mass Spectrometry Network through PROEQUIPRAM program, M14-20 M1420-01-0145-FEDER-000008.
4. Giglio RV, Patti AM, Cicero AFG, Lippi G, Rizzo M, Toth $\mathrm{PP}$, et al. Polyphenols: potential use in the prevention and treatment of cardiovascular diseases. Curr Pharm Des. (2018) 24:239-58. doi: 10.2174/1381612824666180130112652

5. Luca SV, Macovei I, Bujor A, Miron A, Skalicka-Wozniak K, Aprotosoaie AC, et al. Bioactivity of dietary polyphenols: The role of metabolites. Crit Rev Food Sci Nutr. (2020) 60:626-59. doi: 10.1080/10408398.2018.1546669

6. Isemura M, Timmermann BN. Plant polyphenols and health benefitts. Curr Pharm Des. (2013) 19:6051-2. doi: 10.2174/1381612811319340001

7. De Araujo FF, De Paulo Farias D, Neri-Numa IA, Pastore GM. Polyphenols and their applications: An approach in 
food chemistry and innovation potential. Food Chem. (2021) 338:127535. doi: 10.1016/j.foodchem.2020.127535

8. Di Lorenzo C, Colombo F, Biella S, Stockley C, Restani P. Polyphenols and human health: the role of bioavailability. Nutrients. (2021) 13:273. doi: $10.3390 /$ nu13010273

9. Khan N, Mukhtar H. Tea polyphenols in promotion of human health. Nutrients. (2018) 11:39. doi: 10.3390/nu11010039

10. Durazzo A, Lucarini M, Souto EB, Cicala C, Caiazzo E, Izzo AA, et al. Polyphenols: A concise overview on the chemistry, occurrence, human health. Phytother Res. (2019) 33:2221-43. doi: 10.1002/ptr.6419

11. Singla RK, Dubey AK, Garg A, Sharma RK, Fiorino M, Ameen SM, et al. Natural polyphenols: chemical classification, definition of classes, subcategories, and structures. J AOAC Int. (2019) 102:1397400. doi: 10.5740/jaoacint.19-0133

12. Ford KL, Jorgenson DJ, Landry EJL, Whiting SJ. Vitamin and mineral supplement use in medically complex, community-living, older adults. Appl Physiol Nutr Metab. (2019) 44:450-3. doi: 10.1139/apnm-2018-0515

13. Mattea F, Martin A, Cocero MJ. Carotenoid processing with supercritical fluids. J Food Eng. (2009) 93:255-65. doi: 10.1016/j.jfoodeng.2009.01.030

14. Zakynthinos G, Varzakas T. Carotenoids: from plants to food industry. Curr Res Nutr Food Sci. (2016) 4:38-51. doi: 10.12944/CRNFSJ.4.Special-Issue1.04

15. Wu J, Cho E, Willett WC, Sastry SM, Schaumberg DA. Intakes of lutein, zeaxanthin, and other carotenoids and age-related macular degeneration during 2 decades of prospective follow-up. JAMA Ophthalmol. (2015) 133:1415-24. doi: 10.1001/jamaophthalmol.2015.3590

16. Nagarajan J, Ramanan RN, Raghunandan ME, Galanakis CM, Krishnamurthy NP. Carotenoids. In: Galanakis CM, editor. Nutraceutical and Functional Food Components. Academic Press (2017), 259-296. doi: 10.1016/B978-0-12-805257-0.00008-9

17. Eggersdorfer M, Wyss A. Carotenoids in human nutrition and health. Arch Biochem Biophys. (2018) 652:18-26. doi: 10.1016/j.abb.2018.06.001

18. Prieto MA, Lopez CJ, Simal-Gandara J. Glucosinolates: Molecular structure, breakdown, genetic, bioavailability, properties and healthy and adverse effects. Adv Food Nutr Res. (2019) 90:305-50. doi: 10.1016/bs.afnr.2019.02.008

19. Nguyen VPT, Stewart J, Lopez M, Ioannou I, Allais F. Glucosinolates: natural occurrence, biosynthesis, accessibility, isolation, structures, biological activities. Molecules. (2020) 25:4537. doi: 10.3390/molecules 25194537

20. Barba FJ, Nikmaram N, Roohinejad S, Khelfa A, Zhu Z, Koubaa M. Bioavailability of glucosinolates and their breakdown products: impact of processing. Front Nutr. (2016) 3:24. doi: 10.3389/fnut.2016.00024

21. Spagnuolo C, Napolitano M, Tedesco I, Moccia S, Milito A, Russo GL. Neuroprotective role of natural polyphenols. Curr Top Med Chem. (2016) 16:1943-50. doi: 10.2174/1568026616666160204122449

22. Cremonini AL, Caffa I, Cea M, Nencioni A, Odetti P, Monacelli F. Nutrients in the prevention of Alzheimer's disease. Oxid Med Cell Longev. (2019) 2019:9874159. doi: 10.1155/2019/9874159

23. Sarubbo F, Esteban S, Miralles A, Moranta D. Effects of resveratrol and other polyphenols on Sirt1: relevance to brain function during aging. Curr Neuropharmacol. (2018) 16:126-36. doi: 10.2174/1570159X15666170703113212

24. Shankar E, Kanwal R, Candamo M, Gupta S. Dietary phytochemicals as epigenetic modifiers in cancer: Promise and challenges. Semin Cancer Biol. (2016) 40-41:82-99. doi: 10.1016/j.semcancer.2016.04.002

25. Srivastava SK, Arora S, Averett C, Singh S, Singh AP. Modulation of microRNAs by phytochemicals in cancer: underlying mechanisms and translational significance. Biomed Res Int. (2015) 2015:848710. doi: $10.1155 / 2015 / 848710$

26. Tome-Carneiro J, Visioli F. Polyphenol-based nutraceuticals for the prevention and treatment of cardiovascular disease: Review of human evidence. Phytomedicine. (2016) 23:114574. doi: 10.1016/j.phymed.2015.10.018

27. Lopez-Alarcon C, Denicola A. Evaluating the antioxidant capacity of natural products: a review on chemical and cellular-based assays. Anal Chim Acta. (2013) 763:1-10. doi: 10.1016/j.aca.2012.11.051

28. Ilyasov IR, Beloborodov VL, Selivanova IA, Terekhov RP. ABTS/PP decolorization assay of antioxidant capacity reaction pathways. Int J Mol Sci. (2020) 21:1131. doi: 10.3390/ijms21031131
29. Cacciola F, Donato P, Sciarrone D, Dugo P, Mondello L. Comprehensive liquid chromatography and other liquid-based comprehensive techniques coupled to mass spectrometry in food analysis. Anal Chem. (2017) 89:41429. doi: 10.1021/acs.analchem.6b04370

30. Dymerski T. Two-dimensional gas chromatography coupled with mass spectrometry in food analysis. Crit Rev Anal Chem. (2018) 48:25278. doi: 10.1080/10408347.2017.1411248

31. Otles S, Yalcin B. Review on the application of nanobiosensors in food analysis. Acta Sci Pol Technol Aliment. (2012) 11:7-18.

32. Gomez-Arribas LN, Benito-Pena E, Hurtado-Sanchez MDC, Moreno-Bondi MC. Biosensing based on nanoparticles for food allergens detection. Sensors. (2018) 18:1087. doi: 10.3390/s18041087

33. Casado N, Morante-Zarcero S, Perez-Quintanilla D, Camara JS, Sierra I. Two novel strategies in food sample preparation for the analysis of dietary polyphenols: Micro-extraction techniques and new silicabased sorbent materials. Trends Food Sci Technol. (2020) 98:16780. doi: 10.1016/j.tifs.2018.06.020

34. Speltini A, Scalabrini A, Maraschi F, Sturini M, Profumo A. Newest applications of molecularly imprinted polymers for extraction of contaminants from environmental and food matrices: A review. Anal Chim Acta. (2017) 974:1-26. doi: 10.1016/j.aca.2017.04.042

35. Sierra I, Morante-Zarcero S. New advances in food sample preparation with nanomaterials for organic contaminants analysis by liquid chromatography. In: Hussain CM, editor. Nanomaterials in Chromatography. Elsevier (2018), 118-154. doi: 10.1016/B978-0-12-812792-6.00005-4

36. Augusto F, Carasek E, Silva RG, Rivellino SR, Batista AD, Martendal E. New sorbents for extraction and microextraction techniques. J Chromatogr A. (2010) 1217:2533-42. doi: 10.1016/j.chroma.2009.12.033

37. Ötleş S. Handbook of Food Analysis Instruments. New York, NY: CRC Press (2008).

38. Li J, Wang YB, Li KY, Cao YQ, Wu S, Wu L. Advances in different configurations of solid-phase microextraction and their applications in food and environmental analysis. TrAC Trends Analyt Chem. (2015) 72:14152. doi: 10.1016/j.trac.2015.04.023

39. Uwineza PA, Waskiewicz A. Recent advances in supercritical fluid extraction of natural bioactive compounds from natural plant materials. Molecules. (2020) 25:3847. doi: 10.3390/molecules25173847

40. Gonzalez-Salamo J, Socas-Rodriguez B, Hernandez-Borges J, RodriguezDelgado MA. Nanomaterials as sorbents for food sample analysis. Trac-Trends Analyt Chem. (2016) 85:203-20. doi: 10.1016/j.trac.2016. 09.009

41. Casado N, Pérez-Quintanilla D, Morante-Zarcero S, Sierra I. Current development and applications of ordered mesoporous silicas and other solgel silica-based materials in food sample preparation for xenobiotics analysis. TrAC Trends Analyt Chem. (2017) 88:167-84. doi: 10.1016/j.trac.2017. 01.001

42. Casado N, Ganan J, Morante-Zarcero S, Sierra I. New advanced materials and sorbent-based microextraction techniques as strategies in sample preparation to improve the determination of natural toxins in food samples. Molecules. (2020) 25:702. doi: 10.3390/molecules25030702

43. Filippou O, Bitas D, Samanidou V. Green approaches in sample preparation of bioanalytical samples prior to chromatographic analysis. J Chromatogr B Analyt Technol Biomed Life Sci. (2017) 1043:4462. doi: 10.1016/j.jchromb.2016.08.040

44. Armenta S, Garrigues S, De La Guardia M. The role of green extraction techniques in Green Analytical Chemistry. Trac-Trends Analyt Chem. (2015) 71:2-8. doi: 10.1016/j.trac.2014.12.011

45. Cao W, Ye LH, Cao J, Xu JJ, Peng LQ, Zhu QY, et al. Quantitative analysis of flavanones from citrus fruits by using mesoporous molecular sieve-based miniaturized solid phase extraction coupled to ultrahigh-performance liquid chromatography and quadrupole time-of-flight mass spectrometry. J Chromatogr A. (2015) 1406:68-77. doi: 10.1016/j.chroma.2015. 06.035

46. Soltani R, Shahvar A, Dinari M, Saraji M. Environmentally-friendly and ultrasonic-assisted preparation of two-dimensional ultrathin $\mathrm{Ni} / \mathrm{Co}-\mathrm{NO} 3$ layered double hydroxide nanosheet for micro solid-phase extraction of phenolic acids from fruit juices. Ultrason Sonochem. (2018) 40:395401. doi: 10.1016/j.ultsonch.2017.07.031 
47. Peng LQ, Li Q, Chang YX, An M, Yang R, Tan Z, et al. Determination of natural phenols in olive fruits by chitosan assisted matrix solidphase dispersion microextraction and ultrahigh performance liquid chromatography with quadrupole time-of-flight tandem mass spectrometry. J Chromatogr A. (2016) 1456:68-76. doi: 10.1016/j.chroma.2016.06.011

48. Majidi SM, Hadjmohammadi MR. Development of magnetic dispersive micro-solid phase extraction based on magnetic agarose nanoparticles and deep eutectic solvents for the isolation and pre-concentration of three flavonoids in edible natural samples. Talanta. (2021) 222:121649. doi: 10.1016/j.talanta.2020.121649

49. Anastassiades M, Lehotay SJ, Štajnbaher D, Schenck FJ. Fast and easy multiresidue method employing acetonitrile extraction/partitioning and "dispersive solid-phase extraction" for the determination of pesticide residues in produce. J AOAC Int. (2003) 86:41231. doi: $10.1093 /$ jaoac/86.2.412

50. Casado N, Perestrelo R, Silva CL, Sierra I, Câmara JS. An improved and miniaturized analytical strategy based on $\mu$-QuEChERS for isolation of polyphenols. A powerful approach for quality control of baby foods. Microchem J. (2018) 139:110-8. doi: 10.1016/j.microc.2018.02.026

51. Liu H, An K, Su S, Yu Y, Wu J, Xiao G, et al. Aromatic characterization of mangoes (Mangifera indica L.) using solid phase extraction coupled with gas chromatography-mass spectrometry and olfactometry and sensory analyses. Foods. (2020) 9:75. doi: 10.3390/foods 9010075

52. Figueira JA, Porto-Figueira P, Pereira JM, Camara JS. Tangerines cultivated on madeira island-a high throughput natural source of bioactive compounds. Foods. (2020) 9:1470. doi: 10.3390/foods9101470

53. Wang N, Xin H, Zhang Q, Jiang Y, Wang X, Shou D, et al. Carbon nanotubepolymer composite for effervescent pipette tip solid phase microextraction of alkaloids and flavonoids from Epimedii herba in biological samples. Talanta. (2017) 162:10-8. doi: 10.1016/j.talanta.2016.09.059

54. Aresta A, Cotugno P, Massari F, Zambonin C. Determination of transresveratrol in wines, spirits, and grape juices using solid-phase micro extraction coupled to liquid chromatography with UV diode-array detection. Food Anal Methods. (2018) 11:426-31. doi: 10.1007/s12161-017-1013-0

55. Pei M, Huang XJ. Determination of trace phenolic acids in fruit juice samples using multiple monolithic fiber solid-phase microextraction coupled with high-performance liquid chromatography. Analytical Methods. (2016) 8:3831-8. doi: 10.1039/C6AY00934D

56. Figueira JA, Porto-Figueira P, Pereira JM, Camara JS. A comprehensive methodology based on NTME/GC-MS data and chemometric tools for lemons discrimination according to geographical origin. Microchem J. (2020) 157:104933. doi: 10.1016/j.microc.2020.104933

57. Wang N, Huang X, Wang X, Zhang Y, Wu R, Shou D. Pipette tip solid-phase extraction and high-performance liquid chromatography for the determination of flavonoids from Epimedii herba in rat serum and application of the technique to pharmacokinetic studies. J Chromatogr B Analyt Technol Biomed Life Sci. (2015) 990:64-72. doi: 10.1016/j.jchromb.2015.03.012

58. Hao Y, Gao R, Liu D, Tang Y, Guo Z. Selective extraction of gallic acid in pomegranate rind using surface imprinting polymers over magnetic carbon nanotubes. Anal Bioanal Chem. (2015) 407:768190. doi: 10.1007/s00216-015-8930-9

59. Hao Y, Gao R, Liu D, He G, Tang Y, Guo Z. Selective extraction and determination of chlorogenic acid in fruit juices using hydrophilic magnetic imprinted nanoparticles. Food Chem. (2016) 200:215-22. doi: 10.1016/j.foodchem.2016.01.004

60. Wu JR, Xiao DL, Zhao HY, He H, Peng J, Wang CX, et al. A nanocomposite consisting of graphene oxide and $\mathrm{Fe} 3 \mathrm{O} 4$ magnetic nanoparticles for the extraction of flavonoids from tea, wine and urine samples. Microchimica Acta. (2015) 182:2299-306. doi: 10.1007/s00604-015-1575-8

61. Arabi M, Ghaedi M, Ostovan A. Synthesis and application of insitu molecularly imprinted silica monolithic in pipette-tip solid-phase microextraction for the separation and determination of gallic acid in orange juice samples. J Chromatogr B Analyt Technol Biomed Life Sci. (2017) 1048:102-10. doi: 10.1016/j.jchromb.2017.02.016

62. Wang L, Yan H, Yang C, Li Z, Qiao F. Synthesis of mimic molecularly imprinted ordered mesoporous silica adsorbent by thermally reversible semicovalent approach for pipette-tip solid-phase extraction-liquid chromatography fluorescence determination of estradiol in milk. $J$ Chromatogr A. (2016) 1456:58-67. doi: 10.1016/j.chroma.2016.06.010

63. Jiang H, Zhang W, Yang J, Xue G, Su S, Li C, et al. Miniaturized solidphase extraction using a mesoporous molecular sieve SBA-15 as sorbent for the determination of triterpenoid saponins from Pulsatilla chinensis by ultrahigh-performance liquid chromatography-charged aerosol detection. $J$ Pharm Biomed Anal. (2021) 194:113810. doi: 10.1016/j.jpba.2020.113810

64. Silva P, Silva CL, Perestrelo R, Nunes FM, Camara JS. A useful strategy based on chromatographic data combined with quality-by-design approach for food analysis applications. The case study of furanic derivatives in sugarcane honey. J Chromatogr A. (2017) 1520:11726. doi: 10.1016/j.chroma.2017.09.019

65. Teixeira LS, Silva CF, De Oliveira HL, Dinali LF, Nascimento CS, Borges KB. Microextraction by packed molecularly imprinted polymer to selectively determine caffeine in soft and energy drinks. Microchem J. (2020) 158:105252. doi: 10.1016/j.microc.2020.105252

66. Casado N, Perestrelo R, Silva CL, Sierra I, Camara JS. Comparison of high-throughput microextraction techniques, MEPS and muSPEed, for the determination of polyphenols in baby food by ultrahigh pressure liquid chromatography. Food Chem. (2019) 292:14-23. doi: 10.1016/j.foodchem.2019.04.038

67. Porto-Figueira P, Figueira JA, Pereira JA, Camara JS. A fast and innovative microextraction technique, muSPEed, followed by ultrahigh performance liquid chromatography for the analysis of phenolic compounds in teas. $J$ Chromatogr A. (2015) 1424:1-9. doi: 10.1016/j.chroma.2015.10.063

68. Casado N, Morante-Zarcero S, Perez-Quintanilla D, Camara JS, Sierra I. Dispersive solid-phase extraction of polyphenols from juice and smoothie samples using hybrid mesostructured silica followed by ultra-highperformance liquid chromatography-ion-trap tandem mass spectrometry. $J$ Agric Food Chem. (2019) 67:955-67. doi: 10.1021/acs.jafc.8b05578

69. Zou Y, Wang L, Sun H, Wang G, Meng LY, Quinto M, et al. Nanoconfined liquid phase nanoextraction based on carbon nanofibers. Anal Chem. (2021) 93:1310-6. doi: 10.1021/acs.analchem.0c01462

70. Majidi SM, Hadjmohammadi MR. Alcohol-based deep eutectic solvent as a carrier of $\mathrm{SiO} 2 @ \mathrm{Fe} 3 \mathrm{O} 4$ for the development of magnetic dispersive micro-solid-phase extraction method: Application for the preconcentration and determination of morin in apple and grape juices, diluted and acidic extract of dried onion and green tea infusion samples. J Sep Sci. (2019) 42:2842-50. doi: 10.1002/jssc.201900234

71. Peng LQ, Yi L, Yang QC, Cao J, Du LJ, Zhang QD. Graphene nanoplatelets based matrix solid-phase dispersion microextraction for phenolic acids by ultrahigh performance liquid chromatography with electrochemical detection. Sci Rep. (2017) 7:7496. doi: 10.1038/s41598-017-07840-2

72. Xu JJ, Yang R, Ye LH, Cao J, Cao W, Hu SS, et al. Application of ionic liquids for elution of bioactive flavonoid glycosides from lime fruit by miniaturized matrix solid-phase dispersion. Food Chem. (2016) 204:16775. doi: 10.1016/j.foodchem.2016.02.012

73. Du KZ, Chen Y, Li J, Tang G, Tian F, He J, et al. Quantification of eight active ingredients in crude and processed radix polygoni multiflori applying miniaturized matrix solid-phase dispersion microextraction followed by UHPLC. J Sep Sci. (2018) 41:3486-95. doi: 10.1002/jssc.201800342

74. Ding M, Bai Y, Li J, Yang X, Wang H, Gao X, et al. A diol-basedmatrix solid-phase dispersion method for the simultaneous extraction and determination of 13 compounds from angelicae pubescentis radix by ultra high-performance liquid chromatography. Front Pharmacol. (2019) 10:227. doi: 10.3389/fphar.2019.00227

75. Zheng H, Zhen XT, Chen Y, Zhu SC, Ye LH, Yang SW, et al. In situ antioxidation-assisted matrix solid-phase dispersion microextraction and discrimination of chiral flavonoids from citrus fruit via ion mobility quadrupole time-of-flight high-resolution mass spectrometry. Food Chem. (2021) 343:128422. doi: 10.1016/j.foodchem.2020.1 28422

76. Zhang R, Xu N, Wang Y, Liu X, Wang S, Cao J. Metal-organic framework assisted matrix solid-phase dispersion microextraction of saponins using response surface methodology. Electrophoresis. (2020) 41:1354-63. doi: 10.1002/elps.202000042

77. Atlabachew M, Abebe A, Alemneh Wubieneh T, Tefera Habtemariam $\mathrm{Y}$. Rapid and simultaneous determination of trigonelline, caffeine, and 
chlorogenic acid in green coffee bean extract. Food Sci Nutr. (2021) 9:502835. doi: $10.1002 / f s n 3.2456$

78. Magiera S, Kwietniowska E. Fast, simple and efficient salting-out assisted liquid-liquid extraction of naringenin from fruit juice samples prior to their enantioselective determination by liquid chromatography. Food Chem. (2016) 211:227-34. doi: 10.1016/j.foodchem.2016.05.045

79. Park HJ, Jung MY. One step salting-out assisted liquid-liquid extraction followed by UHPLC-ESI-MS/MS for the analysis of isoflavones in soy milk. Food Chem. (2017) 229:797-804. doi: 10.1016/j.foodchem.2017.02.145

80. Zhang Y, Chen X, Li J, Hu S, Wang R, Bai X. Salt-assisted dispersive liquid-liquid microextraction for enhancing the concentration of matrine alkaloids in traditional Chinese medicine and its preparations. J Sep Sci. (2018) 41:3590-7. doi: 10.1002/jssc.201701504

81. Amini T, Hashemi P. Preconcentration and GC-MS determination of caffeine in tea and coffee using homogeneous liquid-liquid microextraction based on solvents volume ratio alteration. J Chromatogr B Analyt Technol Biomed Life Sci. (2018) 1092:252-7. doi: 10.1016/j.jchromb.2018.06.020

82. Shalash M, Makahleh A, Salhimi SM, Saad B. Vortex-assisted liquid-liquidliquid microextraction followed by high performance liquid chromatography for the simultaneous determination of fourteen phenolic acids in honey, iced tea and canned coffee drinks. Talanta. (2017) 174:42835. doi: 10.1016/j.talanta.2017.06.039

83. Fernandez E, Vidal L, Canals A. Rapid determination of hydrophilic phenols in olive oil by vortex-assisted reversed-phase dispersive liquidliquid microextraction and screen-printed carbon electrodes. Talanta. (2018) 181:44-51. doi: 10.1016/j.talanta.2017.12.075

84. Al-Nidawi M, Alshana U, Caleb J, Hassan M, Rahman ZU, Hanoglu DY, et al. Switchable-hydrophilicity solvent liquid-liquid microextraction versus dispersive liquid-liquid microextraction prior to HPLC-UV for the determination and isolation of piperine from Piper nigrum L. J Sep Sci. (2020) 43:3053-60. doi: 10.1002/jssc.202000152

85. Ismaili A, Heydari R, Rezaeepour R. Monitoring the oleuropein content of olive leaves and fruits using ultrasound- and salt-assisted liquid-liquid extraction optimized by response surface methodology and high-performance liquid chromatography. J Sep Sci. (2016) 39:405-11. doi: 10.1002/jssc.201500795

86. Wang $\mathrm{M}$, Wang J, Zhang $\mathrm{Y}$, Xia Q, Bi W, Yang X, et al. Fast environment-friendly ball mill-assisted deep eutectic solventbased extraction of natural products. J Chromatogr A. (2016) 1443:262-6. doi: 10.1016/j.chroma.2016.03.061

87. Shishov A, Volodina N, Nechaeva D, Gagarinova S, Bulatov A. An automated homogeneous liquid-liquid microextraction based on deep eutectic solvent for the HPLC-UV determination of caffeine in beverages. Microchem J. (2019) 144:469-73. doi: 10.1016/j.microc.2018.10.014

88. Altunay N, Elik A, Unal Y, Kaya S. Optimization of an ultrasound-assisted alcohol-based deep eutectic solvent dispersive liquid-phase microextraction for separation and preconcentration of quercetin in wine and food samples with response surface methodology. J Sep Sci. (2021) 44:19982005. doi: $10.1002 /$ jssc.202100048

89. Li P, Ye JF, Zhang Y, Wang ZQ, Ren SJ, Li XW, et al. Centrifugation free and air-assisted liquid-liquid microextraction based on deep eutectic solvent for determination of rare ginsenosides in Kang'ai injection. Microchem J. (2018) 142:313-20. doi: 10.1016/j.microc.2018.07.012

90. Aydin F, Yilmaz E, Soylak M. Vortex assisted deep eutectic solvent (DES)-emulsification liquid-liquid microextraction of trace curcumin in food and herbal tea samples. Food Chem. (2018) 243:442-7. doi: 10.1016/j.foodchem.2017.09.154

91. Ferrone V, Genovese S, Carlucci M, Tiecco M, Germani R, Preziuso F, et al. A green deep eutectic solvent dispersive liquid-liquid micro-extraction (DES-DLLME) for the UHPLC-PDA determination of oxyprenylated phenylpropanoids in olive, soy, peanuts, corn, sunflower oil. Food Chem. (2018) 245:578-85. doi: 10.1016/j.foodchem.2017.10.135

92. Homaie O, Afshar Mogaddam MR, Tamizi E, Nemati M. Comparison of organic and deep eutectic solvents based dispersive liquid-liquid microextraction for the analysis of phytosterols in cow milk combined with high-performance liquid chromatography-ultraviolet detector. J Sep Sci. (2021) 44:4167-80. doi: 10.1002/jssc.202100395
93. Bakirtzi C, Triantafyllidou K, Makris DP. Novel lactic acid-based natural deep eutectic solvents: Efficiency in the ultrasound-assisted extraction of antioxidant polyphenols from common native Greek medicinal plants. J Appl Res Med Aromatic Plants. (2016) 3:120-7. doi: 10.1016/j.jarmap.2016.03.003

94. Dai Y, Rozema E, Verpoorte R, Choi YH. Application of natural deep eutectic solvents to the extraction of anthocyanins from Catharanthus roseus with high extractability and stability replacing conventional organic solvents. $J$ Chromatogr A. (2016) 1434:50-6. doi: 10.1016/j.chroma.2016.01.037

95. Mocan A, Diuzheva A, Carradori S, Andruch V, Massafra C, Moldovan C, et al. Development of novel techniques to extract phenolic compounds from Romanian cultivars of Prunus domestica L. and their biological properties. Food Chem Toxicol. (2018) 119:189-98. doi: 10.1016/j.fct.2018.04.045

96. Diuzheva A, Carradori S, Andruch V, Locatelli M, De Luca E, Tiecco $\mathrm{M}$, et al. Use of innovative (micro)extraction techniques to characterise harpagophytum procumbens root and its commercial food supplements. Phytochem Anal. (2018) 29:233-41. doi: 10.1002/pca.2737

97. Sun J, Zhao XE, Dang J, Sun X, Zheng L, You J, et al. Rapid and sensitive determination of phytosterols in functional foods and medicinal herbs by using UHPLC-MS/MS with microwave-assisted derivatization combined with dual ultrasound-assisted dispersive liquid-liquid microextraction. J Sep Sci. (2017) 40:725-32. doi: 10.1002/jssc.201600711

98. Quigley A, Walsh SW, Hayes E, Connolly D, Cummins W. Effect of seaweed supplementation on tocopherol concentrations in bovine milk using dispersive liquid-liquid microextraction. J Chromatogr B Analyt Technol Biomed Life Sci. (2018) 1092:152-7. doi: 10.1016/j.jchromb.2018.06.013

99. Ramirez DA, Locatelli DA, Torres-Palazzolo CA, Altamirano JC, Camargo AB. Development of garlic bioactive compounds analytical methodology based on liquid phase microextraction using response surface design. Implications for dual analysis: Cooked and biological fluids samples. Food Chem. (2017) 215:493-500. doi: 10.1016/j.foodchem.2016.07.170

100. Ramirez DA, Altamirano JC, Camargo AB. Multi-phytochemical determination of polar and non-polar garlic bioactive compounds in different food and nutraceutical preparations. Food Chem. (2021) 337:127648. doi: 10.1016/j.foodchem.2020.127648

101. Viegas O, Esteves C, Rocha J, Melo A, Ferreira I. Simultaneous determination of melatonin and trans-resveratrol in wine by dispersive liquid-liquid microextraction followed by HPLC-FLD. Food Chem. (2021) 339:128091. doi: 10.1016/j.foodchem.2020.128091

102. Frizzarin RM, Maya F, Estela JM, Cerda V. Fully-automated in-syringe dispersive liquid-liquid microextraction for the determination of caffeine in coffee beverages. Food Chem. (2016) 212:759-67. doi: 10.1016/j.foodchem.2016.06.032

103. Yousefi S, Kamankesh M, Jazaeri S, Attaran A, Mohammadi A. A simple, effective and highly sensitive analytical method used for the determination of caffeine in tea and energy drink samples, and method optimization using a central composite design. Analytical Methods. (2017) 9:166571. doi: 10.1039/C6AY03490J

104. Triaux Z, Petitjean H, Marchioni E, Boltoeva M, Marcic C. Deep eutectic solvent-based headspace single-drop microextraction for the quantification of terpenes in spices. Anal Bioanal Chem. (2020) 412:93348. doi: 10.1007/s00216-019-02317-9

105. Jahromi Z, Mostafavi A, Shamspur T, Mohamadim M. Magnetic ionic liquid assisted single-drop microextraction of ascorbic acid before its voltammetric determination. J Sep Sci. (2017) 40:4041-9. doi: 10.1002/jssc.2017 00664

106. Ji S, Wang Y, Su Z, He D, Du Y, Guo M, et al. Ionic liquids-ultrasound based efficient extraction of flavonoid glycosides and triterpenoid saponins from licorice. RSC Adv. (2018) 8:13989-96. doi: 10.1039/C8RA01056K

107. Chaharlangi M, Parastar H, Malekpour A. Analysis of bioactive constituents of saffron using ultrasonic assisted emulsification microextraction combined with high-performance liquid chromatography with diode array detector: a chemometric study. RSC Adv. (2015) 5:26246-54. doi: 10.1039/C5RA00488H

108. Ranjbar Banforuzi S, Hadjmohammadi MR. Two-phase hollow fiberliquid microextraction based on reverse micelle for the determination of quercetin in human plasma and vegetables samples. Talanta. (2017) 173:1421. doi: 10.1016/j.talanta.2017.05.058 
109. Li MM, Hu S, Chen X, Bai XH. Development of a novel hollow-fiber liquid-phase microextraction based on oil-in-salt and its comparison with conventional one. J Sep Sci. (2017) 40:2941-9. doi: 10.1002/jssc.201700481

110. Zhang SM, Zhang XX, Chen X, Hu S, Bai XH. Deep eutectic solventbased hollow fiber liquid-phase microextraction for quantification of Qmarkers of cinnamic acid derivatives in traditional Chinese medicines and research of their plasma protein binding rates. Microchem J. (2020) 155:104696. doi: 10.1016/j.microc.2020.104696

111. Gao P, Chen $\mathrm{X}, \mathrm{Hu} \mathrm{S}$, Bai X, Luo $\mathrm{M}$. New oil-in-salt liquidphase microextraction on permutite for the extraction and concentration of alkaloids in Coptis chinensis. J Sep Sci. (2017) 40:1334-42. doi: 10.1002/jssc.201601126

112. Wang RQ, Ge X, Chen X, Hu S, Yang L, Li D, et al. Ballpoint tipprotected oil-in-salt liquid-phase microextraction with high performance liquid chromatography for the determination of magnolol and honokiol from cortex Magnoliae officinalis. Instrum Sci Technol. (2019) 48:254268. doi: 10.1080/10739149.2019.1705480

113. Xie LY, Bai J, Zhang XX, Chen X, Bai XH, Hu S. Simultaneous determination of curcuminoids in Curcumae Longae Rhizoma and turmeric tea using liquid-phase microextraction based on solidification of floating deep eutectic solvent drop. Microchem J. (2020) 159:105341. doi: 10.1016/j.microc.2020.105341

114. Chen X, Li J, Hu S, Bai X, Zhao H, Zhang Y. Fibroin/dodecanol floating solidification microextraction for the preconcentration of trace levels of flavonoids in complex matrix samples. J Chromatogr B Analyt Technol Biomed Life Sci. (2018) 1072:17-24. doi: 10.1016/j.jchromb.2017.09.023

115. Hu S, Yang X, Xue J, Chen X, Bai XH, Yu ZH. Graphene/dodecanol floating solidification microextraction for the preconcentration of trace levels of cinnamic acid derivatives in traditional Chinese medicines. J Sep Sci. (2017) 40:2959-66. doi: 10.1002/jssc.201700169

116. Khajeh M, Fard S, Bohlooli M, Ghaffari-Moghaddam M, Khatibi A. Extraction of caffeine and gallic acid from coffee by electrokinetic methods coupled with a hollow-fiber membrane. J Food Process Eng. (2017) 40:e12565. doi: 10.1111/jfpe.12565

117. Trefz P, Rosner L, Hein D, Schubert JK, Miekisch W. Evaluation of needle trap micro-extraction and automatic alveolar sampling for point-of-care breath analysis. Anal Bioanal Chem. (2013) 405:310515. doi: 10.1007/s00216-013-6781-9

118. Mirnaghi FS, Mousavi F, Rocha SM, Pawliszyn J. Automated determination of phenolic compounds in wine, berry, and grape samples using 96-blade solid phase microextraction system coupled with liquid chromatography-tandem mass spectrometry. J Chromatogr A. (2013) 1276:12-9. doi: 10.1016/j.chroma.2012.12.043
119. Choubey S, Goyal S, Varughese LR, Kumar V, Sharma AK, Beniwal V. Probing gallic acid for its broad spectrum applications. Mini Rev Med Chem. (2018) 18:1283-93. doi: 10.2174/1389557518666180330114010

120. Pereira JM, Goncalves J, Porto-Figueira P, Figueira JA, Alves V, Perestrelo R, et al. Current trends on microextraction by packed sorbent - fundamentals, application fields, innovative improvements and future applications. Analyst. (2019) 144:5048-74. doi: 10.1039/C8AN02464B

121. Kissoudi M, Samanidou V. Recent advances in applications of ionic liquids in miniaturized microextraction techniques. Molecules. (2018) 23:1437. doi: 10.3390/molecules23061437

122. Li X, Row KH. Development of deep eutectic solvents applied in extraction and separation. J Sep Sci. (2016) 39:3505-20. doi: 10.1002/jssc.2016 00633

123. Shishov A, Bulatov A, Locatelli M, Carradori S, Andruch V. Application of deep eutectic solvents in analytical chemistry. A review. Microchem J. (2017) 135:33-8. doi: 10.1016/j.microc.2017.07.015

124. Nie L, Toufouki S, Yao S, Guo D. Rethinking the applications of ionic liquids and deep eutectic solvents in innovative nano-sorbents. Front Chem. (2021) 9:e653238. doi: 10.3389/fchem.2021.653238

125. Hadjmohammadi M, Karimiyan H, Sharifi V. Hollow fibre-based liquid phase microextraction combined with high-performance liquid chromatography for the analysis of flavonoids in Echinophora platyloba DC. and Mentha piperita. Food Chem. (2013) 141:731-5. doi: 10.1016/j.foodchem.2013.02.083

Conflict of Interest: The authors declare that the research was conducted in the absence of any commercial or financial relationships that could be construed as a potential conflict of interest.

Publisher's Note: All claims expressed in this article are solely those of the authors and do not necessarily represent those of their affiliated organizations, or those of the publisher, the editors and the reviewers. Any product that may be evaluated in this article, or claim that may be made by its manufacturer, is not guaranteed or endorsed by the publisher.

Copyright (c) 2022 Pereira, Casado, Porto-Figueira and Câmara. This is an openaccess article distributed under the terms of the Creative Commons Attribution License (CC BY). The use, distribution or reproduction in other forums is permitted, provided the original author(s) and the copyright owner(s) are credited and that the original publication in this journal is cited, in accordance with accepted academic practice. No use, distribution or reproduction is permitted which does not comply with these terms. 


\section{GLOSSARY}

AA, Ascorbic acid; ACN, acetonitrile; APCI-MS, atmosphericpressure chemical ionization mass spectrometry; BIN, barrel insert needle; $[\mathrm{Bmin}] \mathrm{BF}_{4}, \quad$ 1-butyl-3-methylimidazolium tetrafluoroborate; BT-IOS-LPME, Ballpoint tip-protected oilin-salt liquid-phase microextraction; CAD, charged aerosol detector; CNFs, carbon nanofibers; CMPI, 2-Chloro1-Methyl Pyridinium Iodide; CNTs, carbon nanotubes; CSR, 4'-Carboxy-substituted rosamine; DAD, diode-array detection; DCM, dichloromethane; DES, deep eutectic solvents; DLLME- dispersive liquid-liquid microextraction; DMAP, 4dimethylaminopyridine; dSPE, dispersive solid-phase extraction; DVB, divinylbenzene; ECD, electrochemical detection; EPTSPME, effervescent pipette tip solid phase microextraction; EtAc, ethyl acetate; EtOH, ethanol; FA, formic acid; FGOTD-PTFE, functional graphene oxide thermal desorption poly(tetrafluoroethylene); FLD, fluorescence detector; FSME, floating solidification microextraction; GA, gallic acid; GAC, Green Analytical Chemistry; GC-MS, gas chromatographymass spectrometry; HAc, acetic acid; HF-EKE, hollow fiber electrokinetic extraction; HF-LPME, hollow-fiber liquid-phase microextraction; HLB, hydrophilic-lipophilic-balanced; HS, headspace; ILs, ionic liquids; IOS-LPME, oil-in-salt liquidphase microextraction; LC-MS, liquid chromatography mass spectrometry; LDH, layered double hydroxide; LDLs, lowdensity lipoproteins; LE, liquid extraction; LLME, liquid-liquid microextraction; M-ILs-SDME, magnetic ionic liquids single drop microextraction; MIP, molecular imprinted polymer; MIOMS-ir, molecularly imprinted ordered mesoporous silica imprint-removed silica; MISM, molecularly imprinted silica monolithic; ME, microextraction; $\mathrm{MeOH}$, methanol; MEPS, microextraction by packed sorbent; MMF, multiple monolithic fiber; MNPs, magnetic nanoparticles; MOF, Metal-organic framework; MSPDM, matrix solid-phase dispersion microextraction; MWCNTs, Multi-walled CNTs; M-SPE, magnetic solid-phase extraction; NADES, natural deep eutectic solvents; NCS, nanoconfined solvent; NPs, nanoparticles; NTD, needle trap device; NTME, needle-trap microextraction; OS, organic solvents; PDA, photodiode array; PDMS, polydimethylsiloxane; PLE, pressurized liquid extraction; PT, pipette tip; PS/DVB-RP, reverse phase polystyrenedivinylbenzene sorbent; RAMIPs, restricted access molecularly imprinted polymers; RT, room temperature; SALLE, salting-out liquid-liquid extraction; SBA-15, Santa Barbara Amorphous 15 molecular sieve; SHS, Switchable-hydrophilicity solvent; SE, solvent extraction; SPME, solid-phase microextraction; SULLE, sugaring-out assisted liquid-liquid extraction; TCM, traditional Chinese medicine; THF, Tetrahydrofuran; US, ultrasonication; UHPLC, ultrahigh performance liquid chromatography; USAEME, ultrasonic assisted emulsification microextraction; UV, ultraviolet analysis; VA, vortex assisted VOCs, volatile organic compounds; $\mu \mathrm{SPE}$, micro solid-phase extraction. 